\title{
Learning about Consumption Dynamics
}

\author{
Michael Johannes, Lars Lochstoer, and Yiqun Mou* \\ Columbia Business School
}

First draft: March 2010

February 9, 2011

\begin{abstract}
This paper studies the asset pricing implications of Bayesian learning about the parameters, states, and models determining aggregate consumption dynamics. Our approach is empirical and focuses on the quantitative implications of learning in real-time using post World War II consumption data. We characterize this learning process and provide empirical evidence that revisions in beliefs stemming from parameter and model uncertainty are significantly related to realized aggregate equity returns. Further, we show that beliefs regarding the conditional moments of consumption growth are strongly time-varying and exhibit business cycle and/or long-run fluctuations. Much of the long-run behavior is unanticipated ex ante. We embed these subjective beliefs in a general equilibrium model and find that about half of the post World-War II observed equity market risk premium and much of the observed return predictability are due to unexpected revisions in beliefs about parameters and models governing consumption dynamics.
\end{abstract}

*All authors are at Columbia Business School, Department of Finance and Economics. We would like to thank Pierre Collin-Dufresne, Lars Hansen (AFA discussant), and seminar participants at AFA Denver 2011, Columbia University, and the University of Wisconsin (Madison) for helpful comments. Corresponding author: Lars Lochstoer, 405B Uris Hall, 3022 Broadway, New York, NY 10027. Email: LL2609@columbia.edu 


\section{Introduction}

This paper studies the asset pricing implications of learning about aggregate consumption dynamics in the U.S. post-World War II experience. We are motivated by the practical difficulties generated by using increasingly complicated consumption-based asset pricing models with many difficult-to-estimate parameters and latent state variables. For example, parameters and states controlling long-run consumption growth dynamics are at once extremely important for asset pricing and particularly difficult to estimate. Thus, we are interested in studying an economic agent who is burdened with some of the same econometric problems faced by researchers, a problem suggested by Hansen (2007). ${ }^{1}$

A large existing literature studies the asset pricing implications of statistical learningthe process of updating beliefs about uncertain parameters, state variables, or even model specifications. Pastor and Veronesi (2009) provide a recent survey. In theory, learning can generate a wide range of implications relating to stock valuation, levels and variation in expected returns and volatility, and time series predictability, with many of the results focussed on the implications of learning about dividend dynamics.

Our analysis differs from the extant literature along two important dimensions. First, we focus on the empirical implications of simultaneously learning about all parameters, state variables, and model specifications. Nearly all existing work focuses on learning a single unknown parameter or state variable, likely for tractability reasons. In our setting, all of the parameters and states are unknown, and there is additional model uncertainty. Second, we focus on the specific implications of learning in real time from macroeconomic data about consumption dynamics using the U.S. post World War II experience. Thus, we are not specifically interested in asset pricing implications of learning in repeated sampling settings (i.e. in population), but rather the specific implications generated by the macroeconomic shocks realized over the past 60 years in the United States.

By focussing on the post war experience (and not on unconditional implications averaged over potential data sets), we highlight that learning induces non-stationary dynamics. This non-stationarity has long been recognized as potentially important, but little-if anyempirical work has been done to investigate its implications. Lucas and Sargent (1979) note

\footnotetext{
${ }^{1}$ Hansen (2007) states: "In actual decision making, we may be required to learn about moving targets, to make parametric inferences, to compare model performance, or to gauge the importance of long-run components of uncertainty. As the statistical problem that agents confront in our model is made complex, rational expectations' presumed confidence in their knowledge of the probability specification becomes more tenuous. This leads me to ask: (a) how can we burden the investors with some of the specification problems that challenge the econometrician, and (b) when would doing so have important quantitative implications" (p.2).
} 
this, and provide an explanation for why little empirical work has been done:

"it has been only a matter of analytical convenience and not of necessity that equilibrium models have used the assumption of stochastically stationary "shocks" and the assumption that agents have already learned the probability distributions that they face. Both of these assumptions can be abandoned, albeit at a cost in terms of the simplicity of the model.... While models incorporating Bayesian learning and stochastic nonstationarity are both technically feasible and consistent with the equilibrium modeling strategy, almost no successful applied work along these lines has come to light. One reason is probably that nonstationary time series models are cumbersome and come in so many varieties" (p. 68).

This paper addresses these issues, with the goal of quantifying the asset pricing implications of rational learning about consumption growth dynamics. In particular, we focus on the following types of questions. Could an agent who updates his beliefs rationally detect non-i.i.d. consumption growth dynamics in real time? How rapidly does the agent learn about parameters and models? Are the revisions in beliefs about consumption moments correlated with asset returns, as a learning story would require? Is there evidence that the nonstationarities generated by learning help us understand standard asset pricing puzzles, such as the high equity premium, return volatility, and degree of return predictability?

We address these questions in the context of standard discrete-state Markov switching models of consumption growth. We consider three models: unrestricted two and three state models and a two-state model restricted to generate i.i.d. consumption growth. The states capture business cycle frequency fluctuations and can be labeled as expansion and recession in two-state models, with an additional 'disaster' state in three-state models. ${ }^{2}$ Our key assumption is that the agent views the parameters, states, and even models as unknowns, and uses Bayes rule to learn using current and past consumption data in a base case and additional macroeconomic data such as GDP growth in extensions.

\footnotetext{
${ }^{2}$ Rietz (1988) and, more recently, Barro (2006, 2009) argue that consumption disaster risk can help explain some of the standard macro-finance asset pricing puzzles.

A Markov switching model for consumption or dividends is a benchmark specification under the assumption that the states and parameters are observed. These specifications have been widely used, see, e.g., Mehra and Prescott (1985), Rietz (1988), Cecchetti, Lam, and Mark (1990, 1993), Whitelaw (2000), Cagetti, Hansen, Sargent, and Williams (2002), Barro (2006), Barro and Ursua (2008), Chen (2008), Bhamra, Kuehn, and Strebulaev (2008), Barro, Nakamura, Steinsson and Ursua (2009), Backus, Chernov, and Martin (2009), and Gabaix (2009).
} 
To focus on different aspects of learning, we consider three distinct initial beliefs over consumption dynamics. The first, the historical prior, uses Shiller's consumption data from 1889 until 1946 to 'train' the prior distribution, a common approach for generating 'noninformative' priors. ${ }^{3}$ The second, the 'look-ahead' prior, sets prior parameter means to fullsample maximum likelihood point estimates using the post World War II (WW2) sample. We embed substantial uncertainty around these estimates to study the effect of parameter uncertainty. This is often called an 'empirical Bayes' approach. The third, the fixed parameter case, is a rational expectations benchmark where parameter values are fixed at the end-of-sample estimates, thus there is no parameter uncertainty. This allows us to separate the effects of parameter and state uncertainty.

Our first set of results, which are preference free, characterize the agent's beliefs over time. These results cover statistical learning over parameters, states, and models, and the agent's beliefs over future consumption dynamics (e.g., moments). In terms of models, the posterior probability of the i.i.d. model falls dramatically over time regardless of its prior weight, provided it is less than one. Thus our agent learns, in real-time, that consumption growth is not i.i.d., but has persistent components. ${ }^{4}$ The agent believes the conditional mean of consumption growth is low in recessions and high in expansions, with the opposite pattern for consumption growth volatility. The two-state model quickly emerges as the most likely, but the three-state model, which includes a disaster state, still has a probability of $5-10 \%$ at the end of the sample. ${ }^{5}$ At the onset of the financial crisis in 2008, the probability of the disaster model increases.

In terms of the parameters, there is significant learning about the expansion state, slower learning about the recession state, and barely any learning about the disaster state, as it is rarely, if ever, visited. Thus, there is an observed differential in the speed of learning. Parameter learning, as opposed to learning about the cyclical states, generates non-stationary time-variation in the conditional means and variances of consumption growth, as well as measures of non-normality such as skewness and kurtosis. For both the historical and the look-ahead priors (after a 10 year burn-in), the agent's perception of the long-run mean (volatility) of consumption growth increases (decreases) over the sample. Also, the perceived

\footnotetext{
${ }^{3}$ We do account for measurement error, which likely increased reported macroeconomic volatility during the pre-war period, as argued in Romer (1989).

${ }^{4}$ This result is robust to persistence induced by time-aggregation of the consumption data (see Working (1960)).

${ }^{5}$ The posterior probability of the three-state model would change dramatically, if visited. For example, if a $-3 \%$ quarterly consumption growth shock were realized at the end of the sample, the posterior probability of the three-state model would increase to almost $50 \%$.
} 
persistence of recessions decreases, while the perceived persistence of expansions increases. As the agent's beliefs about these parameters and moments change, asset prices and risk premia will also change. Further, these non-stationary revisions in parameter and model beliefs can generate a quantitatively important wedge between ex post outcomes and ex ante beliefs, which we later show helps explain the high sample equity premium as well as the high level of in-sample excess return predictability. ${ }^{6}$

Our first formal test for the importance of learning consists of regressions of contemporaneous excess stock market returns on revisions in beliefs about expected consumption growth. For learning to matter, unexpected revisions in beliefs about expected consumption growth should be reflected in the unexpected component of aggregate equity returns. ${ }^{7} \mathrm{We}$ find strong statistical evidence that this relationship is positive, and the results are, after a burn-in period to alleviate concerns for prior misspecification, roughly the same for both the historical and the look-ahead prior. To disentangle parameter learning from state learning, we include revisions in beliefs generated by the fixed parameter prior as a control in the regressions. The revisions in beliefs obtained using the historical and look-ahead priors remain statistically significant, while the revisions in beliefs about expected consumption growth generated by the models with known parameters are insignificant.

The fact that learning about parameters and models is a statistically significant determinant of asset returns in our sample, confirms our main hypothesis. This conclusion is strengthened if we allow the agent to learn from both consumption and GDP growth. It is important to note that the results are purely based on real-time learning from fundamentals, as our agent does not use any asset price data (e.g., the dividend-price ratio) when forming beliefs. Since the revisions in beliefs obtained from the models with fixed parameters are not statistically insignificant in these regressions, the evidence questions the standard full-information, rational expectations implementation of the standard consumption-based model, at least for the models of consumption dynamics that we consider.

Next, we consider formal asset pricing implications assuming Epstein-Zin utility. We use standard preference parameters (taken from Bansal and Yaron (2004)) and the real-time estimated beliefs of the agent regarding the consumption dynamics as inputs in the pricing

\footnotetext{
${ }^{6}$ All of the results described in the current and previous paragraphs are robust to learning from additional GDP growth data.

${ }^{7}$ The sign of the effect would in a model depend on the elasticity of intertemporal substitution, and also on the other moments that change at the same time (volatility, skewness, kurtosis, etc.). In the model section, we show that this positive relation is consistent with a model with an elasticity of intertemporal substitution greater than 1.
} 
exercise. ${ }^{8}$ We use anticipated utility to price assets (Kreps (1998)), which is the common approach for handling realistic parameter uncertainty in macroeconomics and finance (see, e.g., Piazzesi and Schneider (2010), Cogley and Sargent (2009)). This implies that our agent prices claims at each point in time using current posterior means for the parameters and model probabilities, assuming those values will persist into the indefinite future. ${ }^{9}$ We do account for state uncertainty when pricing. Given prices, we compute realized (from the perspective of our agent) asset returns, price-dividend ratios, and predictability regressions. This generates a completely out-of-sample time series that we can compare to actual realizations.

Our formal asset pricing results provide additional evidence, along multiple dimensions, for the importance of nonstationarities generated by parameter learning. To see this, it is useful to consider first pricing results generated by the fixed parameters prior for a three-state model specification. This specification generates a realized equity premium of about $1.8 \%$ (less than half of the observed value of $4.7 \%$ over the post WW2 period), an equity volatility of $12.4 \%$ (40\% lower than the observed value of $17.1 \%$, a Sharpe ratio of 0.14 (about $50 \%$ lower than the observed Sharpe ratio of 0.27 ), and a price-dividend ratio volatility of $7 \%$ (more than $80 \%$ lower than the observed value of $38 \%$ ). The quarterly sample correlation between the model-generated and the empirical market price-dividend ratio is $25 \%$. Parameter learning uniformly improves all of these statistics. With the historical prior and an information set consisting of historical consumption (consumption and GDP, respectively) data, the equity premium is $3.7 \%(4.4 \%)$, the realized equity volatility is $17.7 \%$ (17.0\%), the Sharpe ratio is $0.21(0.26)$, and the correlation between the model generated and market price-dividend ratio is $34 \%(58 \%)$. The results are, after a burn-in period, similar for the look-ahead prior.

The increase in the realized equity premium and return volatility is due to unexpected revisions in beliefs resulting from the parameter and model learning. In particular, the average annualized ex ante quarterly risk premium is similar across the models at about $1.8 \%$ (slightly higher in the models with historical prior). ${ }^{10}$ This highlights the importance of the

\footnotetext{
${ }^{8}$ We do price a levered consumption claim and introduce idiosyncratic noise to break the perfect relationship between consumption and dividend growth. The dividends are calibrated to match the volatility of dividend growth and the correlation between dividend and consumption growth.

${ }^{9}$ This approach greatly reduces the dimensionality of the pricing problem, which otherwise would be prohibitively large. As an example, for the 3 -state model there are twelve parameters to learn about, each with two hyperparameters (which serve as state variables) to characterize the posteriors. In addition, using the mean parameter values at each point in time means that we are not subject to the technical issues regarding priced parameter uncertainty pointed out by Geweke (2001) and Weitzman (2007).

${ }^{10}$ Recall that parameter and model uncertainty are not priced risks in the anticipated utility framework.
} 
specific time path of beliefs about parameters and models - a form of nonstationarity - for standard asset pricing statistics, at least relative to the fixed parameter, rational expectations benchmark. This also implies, looking forward, that the perceived equity premium is much smaller than the realized equity premium over the post WW2 period. These points are consistent with the results in Cogley and Sargent (2008). ${ }^{11}$

In terms of predictability regressions, the results closely match the data. In particular for the historical and look-ahead priors and for forecasting excess market returns with the lagged log dividend-price ratio, the generated regression coefficients and $R^{2}$ 's are increasing with the forecasting horizon and similar to those found in the data. The fixed parameters case, however, does not deliver significant ex post predictability, although the ex ante risk premium is in fact time-varying in these models as well. The reason is that the time-variation in the risk premium with fixed parameters is too small relative to the volatility of realized returns to result in significant $t$-statistics in the sample we analyze. The intuition for why insample predictability occurs when agents are uncertain about parameters and models is the same as in Timmermann (1993) and Lewellen and Shanken (2002) - unexpected updates in growth and discount rates impact the dividend-price ratio and returns in opposite directions leading to a positive in-sample relation. Thus, in-sample predictability can be expected with parameter and model learning. The quantitatively large degree of in-sample relative to out-of-sample predictability that we document in the model is consistent with the literature. For example, Fama and French (1988) document a high degree of in-sample predictability of excess (long-horizon) stock market returns using the price-dividend ratio as the predictive variable. On the other hand, Goyal and Welch (2008) and Ang and Bekaert (2007) document poor out-of-sample performance of these regressions in the data, and the historical and lookahead prior learning models presented here are consistent with this evidence.

In conclusion, our results strongly support the importance of parameter and model learning for understanding four distinct asset pricing regularities. First, we show that parameter and model learning leads to a specific time path of belief revisions that are correlated with the time series of realized equity returns, controlling for realized consumption growth. Second, the time series of beliefs help explain the time-series of the price level of the market (the

\footnotetext{
${ }^{11}$ Cogley and Sargent (2008) assume negatively biased beliefs about the consumption dynamics to highlight the same mechanism and also consider the role of robustness. In their model, the subjective probability of recessions is higher than the 'objective' estimate from the data. The results we present here are consistent with their conclusions, but our models are estimated from fundamentals in real-time, which allows for an outof-sample examination of the time-series of revisions in beliefs. Further, we allow for learning over different models of the data generating process, as well as all the parameters of each model.
} 
time-series of the price-dividend ratio) in a general equilibrium model. Third, the time series of beliefs display strong non-stationarity that drives a wedge between ex-ante beliefs and expost realizations that is absent in rational expectations models. This helps explain common asset pricing puzzles such as excess return volatility, the high sample equity premium, and the high degree of in-sample return predictability, relative to a fixed parameter alternative. All of these results are generated by real-time learning from consumption and GDP growth, using standard preference parameters without directly calibrating to asset returns. In this sense the results are entirely "out-of-sample."

The rest of the paper proceeds as follows. Section 2 introduces the model, the learning setting, our methodological approach, and discusses related literature. Section 3 presents the statistical results, and Section 4 presents the asset pricing results. Section 5 concludes. We give a more in-depth literature review in the Appendix.

\section{The Environment}

\subsection{Model}

We follow a large literature and assume that an exogenous Markov or regime switching process drives the dynamics of aggregate, real, per capita consumption growth. Log consumption growth, $\Delta c_{t}$, evolves via:

$$
\Delta c_{t}=\mu_{s t}+\sigma_{s_{t}} \varepsilon_{t}
$$

where $\varepsilon_{t}$ are i.i.d. standard normal shocks, $s_{t} \in\{1, \ldots, N\}$ is a discretely-valued Markov state variable, and $\left(\mu_{s_{t}}, \sigma_{s_{t}}^{2}\right)$ are the Markov state-dependent mean and variance of consumption growth. The Markov chain evolves via a $N \times N$ transition matrix $\Pi$ with elements $\pi_{i j}$ such that $\operatorname{Prob}\left[s_{t}=j \mid s_{t-1}=i\right]=\pi_{i j}$, with the restriction that $\sum_{j=1}^{N} \pi_{i j}=1$. The fixed parameters

of the $N$-state model contain the means and variances in each state, $\left\{\mu_{n}, \sigma_{n}^{2}\right\}_{n=1}^{N}$ as well as the elements of the transition matrix. The transition matrix controls the persistence of the Markov state.

Markov switching models are flexible and tractable and have been widely used since Mehra and Prescott (1985) and Rietz (1988). By varying the number, persistence, and distribution of the states, the model can generate a wide range of economically interesting and statistically flexible distributions. Although the $\varepsilon_{t}$ 's are i.i.d. normal and the distribution 
of consumption growth, conditional on the Markov state and parameter values, is normally distributed, the distribution of future consumption growth is neither i.i.d. nor normal due to the time-varying moments generated by the shifting Markov state variable. This timevariation induces very flexible marginal and predictive distributions for consumption growth. These models are also tractable, as it is possible to compute likelihood functions and filtering distributions, given parameters.

We consider two and three state models and also consider a restricted version of the two state model generating i.i.d consumption growth by imposing the restriction $\pi_{11}=\pi_{21}$ and $\pi_{22}=\pi_{12}=1-\pi_{11}$. Under this assumption, consumption growth is an i.i.d. mixture of two normal distributions, essentially a discrete-time version of Merton's (1976) mixture model. The general two and three-state models have 6 and 12 parameters, respectively. The i.i.d. two state model has 5 parameters $\left(\mu_{1}, \mu_{2}, \sigma_{1}, \sigma_{2}\right.$ and $\left.\pi_{11}\right)$.

It is common in these models to provide business cycle labels to the states. In a two-state model, we interpret the two states as 'recession' and 'expansion,' while the three state model additionally allows for a 'disaster' state. ${ }^{12}$ Although rare event models have been used for understanding equity valuation since Rietz (1988), there has been a recent resurgence in research using these models (see, e.g., Barro (2006, 2009), Barro and Ursua (2008), Barro, Nakamura, Steinsson and Ursua (2009), Backus, Chernov, and Martin (2009), and Gabaix (2009)).

\subsection{Information and learning}

To operationalize the model, additional assumptions are required regarding the economic agent's information set. Since we want to model a learning environment akin to that faced by the econometrician, we allow agents to be uncertain about the Markov state, the parameters, and the total number of Markov states. These are called state, parameter, and model uncertainty, respectively. In this paper, we assume agents are Bayesian, which means they update initial beliefs via Bayes' rule as data arrives.

The Bayesian learning problem is as follows. We consider $k=1, \ldots, K$ models, $\left\{\mathcal{M}_{k}\right\}_{k=1}^{K}$, and in model $\mathcal{M}_{k}$, the state variables and parameters are denoted as $s_{t}$ and $\theta$, respec-

\footnotetext{
${ }^{12}$ We do not consider, for instance, 1- or 4-state models as the Likelihood ratios of these relative to the 2or 3-state model show that the 2- and 3-state models better describe the data. As we will show, however, there is some time-variation in whether a 2- or 3-state model matches the data better, which is one of the reasons we entertain both of these as alternative models.
} 
tively. ${ }^{13}$ The distribution $p\left(\theta, s_{t}, \mathcal{M}_{k} \mid y^{t}\right)$ summarizes the uncertainty after observing data $y^{t}=\left(y_{1}, \ldots y_{t}\right)$. To understand the components of the learning problem, we can decompose the posterior as:

$$
p\left(\theta, s_{t}, \mathcal{M}_{k} \mid y^{t}\right)=p\left(\theta, s_{t} \mid \mathcal{M}_{k}, y^{t}\right) p\left(\mathcal{M}_{k} \mid y^{t}\right)
$$

$p\left(\theta, s_{t} \mid \mathcal{M}_{k}, y^{t}\right)$ solves the parameter and state "estimation" problem conditional on a model and $p\left(\mathcal{M}_{k} \mid y^{t}\right)$ provides model probabilities. It is important to note that this is a non-trivial, high-dimensional learning problem, as posterior beliefs depend in a complicated manner on past data and can vary substantially over time. The dimensionality of the posterior can be high, in our case more than 10 dimensions.

One of our primary goals is to characterize and understand the asset pricing implications of the transient process of learning about the parameters, states, and models. ${ }^{14}$ Learning generates a form of non-stationarity, since parameter estimates and model probabilities are changing through the sample. When pricing assets, this can lead to large differences between ex ante beliefs and ex post outcomes, as shown in Cogley and Sargent (2008). Given this non-stationarity, we are concerned with understanding the implications of learning based on the specific experience of the U.S. post-war economy. ${ }^{15}$

To operationalize the learning problem, we need to specify the prior distribution, the data the agent uses to update beliefs, and develop an econometric method for sampling from the posterior distribution. In terms of data, we in a benchmark case assume that agents learn only from observing past and current consumption growth, a common assumption in the learning literature (see, e.g., Cogley and Sargent (2008) and Hansen and Sargent (2009)). The primary data used is the 'standard' dataset consisting of real, per capita quarterly consumption growth observations obtained from the Bureau of Economic Analysis (the National Income and Product Account tables) from 1947:Q1 until 2009:Q1. We develop and implement extensions that allow updating using additional variables such as GDP growth.

\footnotetext{
${ }^{13}$ This is a notational abuse. In general, the state and dimension of the parameter vector should depend on the model, thus we should superscript the parameters and states by ' $k$ ', $\theta^{k}$ and $s_{t}^{k}$. For notational simplicity, we drop the model dependence and denote the parameters and states as $\theta$ and $s_{t}$, respectively.

${ }^{14}$ These type of problems received quite a bit of theoretical attention early in the rational expectations paradigm - see for example Bray and Savin (1986) for a discussion of model specification and convergence to rational expectations equilibria by learning from observed outcomes.

${ }^{15}$ This is different from the standard practice of looking at population or average small-sample unconditional asset price and consumption growth moments from a model calibrated to the U.S. postwar data - we are looking at a single outcome corresponding to the U.S. post-war economy.
} 


\subsection{Initial beliefs}

The learning process begins with initial beliefs or the prior distribution. In terms of functional forms, we assume proper, conjugate prior distributions (Raiffa and Schlaifer (1956)). One alternative would be flat or 'uninformative' priors, but this is not possible, however, in Markov switching models, as this creates identification issues (the label switching problem) and causes problems with algorithms for sampling from the posterior. ${ }^{16}$ Conjugate priors imply that the functional form of beliefs is the same before and after sampling, are analytically tractable for econometric implementation, and are flexible enough to express a wide range initial beliefs.

For the mean and variance parameters in each state, $\left(\mu_{i}, \sigma_{i}^{2}\right)$, the conjugate prior is $p\left(\mu_{i} \mid \sigma_{i}^{2}\right) p\left(\sigma_{i}^{2}\right) \sim \mathcal{N} \mathcal{I} \mathcal{G}\left(a_{i}, A_{i}, b_{i}, B_{i}\right)$, where $\mathcal{N} \mathcal{I} \mathcal{G}$ is the normal/inverse gamma distribution. The transition probabilities are assumed to follow a Beta distribution in two-state specification and its generalization, the Dirichlet distribution, in models with three states. Calibration of the hyperparameters completes the specification.

We endow our agent with economically interesting initial beliefs to study how learning proceeds from various starting points. We consider three prior distributions and use an 'objective' approach to calibrate the prior parameters. The first, the 'historical prior,' uses a training sample to calibrate the prior distribution. Training samples are the most common way of generating non-subjective prior distributions (see, e.g., O'Hagan (1994)). In this case, an initial dataset is used to provide information on the location and scale of the parameters. In our application, we use the annual consumption data from Shiller from 1889 until 1946. Given the prior generated from the training sample, learning proceeds on the second dataset - in our case, the post World War II sample. ${ }^{17}$

The second is called the 'look-ahead prior.' This prior sets the prior mean for each parameter equal to full-sample maximum likelihood estimates using the post World War II sample, similar to the procedure employed in an 'Empirical Bayes' approach. The prior

\footnotetext{
${ }^{16}$ The label switching problem refers to the fact that the likelihood function is invariant to a relabeling of the components. For example, in a two-state model, it is possible to swap the definitions of the first and second states and the associated parameters without changing the value of the likelihood. The solution is to impose parameter constraints in optimization for MLE or to use informative prior distributions for Bayesian approaches. These constraints/information often take the form of an ordering of the means or variances of the parameters. For example in a two state model, it is common to impose that $\mu_{1}<\mu_{2}$ and/or $\sigma_{1}<\sigma_{2}$ to breaks the symmetry of the likelihood function.

${ }^{17}$ Romer (1989) presents evidence that a substantial fraction of the volatility of macro variables such as consumption growth pre-WW2 is due to measurement error. To alleviate this concern, we set the prior mean over the variance parameters to a quarter of the value estimated over the training sample. See the Appendix for further details.
} 
variances are chosen to be relatively flat around these full-sample estimates, in order to allow for meaningful learning about the parameters as new data arrives, without running into label-switching identification problems. This approach violates the central idea of the Bayesian approach, as the prior contains information from the sample, but it is useful for analyzing the evolution of parameter uncertainty through the post World War II sample. The main differences between the historical and the look-ahead priors are that the historical priors have on average higher consumption growth volatility, shorter expansions, and longer recessions. For the three-state model, the disaster state is also more severe in the historical prior, reflecting the Great Depression.

The third is called the 'fixed parameter' prior. This is a point-mass prior located at the end-of-sample estimates. In this case, the agent only learns about the latent Markov state. This prior mimics the typical rational expectations approach and allows us to separately identify the role of state and parameter learning, since the other priors have both state and parameter learning.

The details of the priors, the specific prior parameters chosen, as well as a description of the econometric technique we apply to solve this high-dimensional learning problem (particle filtering) are given in the Appendix.

\section{Statistical results: time-series of subjective beliefs}

We have two main sets of results. The first is purely statistical and does not require any economic assumptions regarding utility or asset pricing. These results are discussed in this section and focus on the time series of the agent's beliefs, and how revisions to these beliefs are related to asset prices. We discuss state, parameter, and model learning and their implication for the time series of conditional consumption moments, as perceived by the Bayesian agent. After summarizing these statistical results, we empirically investigate how revisions in the agent's beliefs are related to stock market returns. We also consider the case of learning from GDP data, in addition to consumption data. The second set of results requires additional preference and pricing assumptions and is discussed in the following section.

\subsection{State and parameter learning}

Conditional on a model specification, our agent learns about the Markov state and the parameters determining consumption dynamics, with revisions in beliefs generated by a 
combination of data, model specification, and initial beliefs. To start, consider the agent's beliefs about the current state of the economy, $s_{t}$, where state 1 is an 'expansion' state, state 2 the 'contraction' state and, if a three-state model, state 3 the 'Disaster' state. Estimates are given by

$$
E\left[s_{t} \mid \mathcal{M}_{k}, y^{t}\right]=\int s_{t} p\left(\theta, s_{t} \mid \mathcal{M}_{k}, y^{t}\right) d \theta d s_{t}
$$

Note that these are marginal mean state beliefs, as parameter uncertainty is integrated out. Although $s_{t}$ is discrete, the mean estimates need not be integer valued. Figure 1 displays the posterior state beliefs over time, for each model and for different priors.

There are a number of notable features of these beliefs. NBER recessions (shaded yellow) and expansions are clearly identified in the models. The only exceptions to this are the recessions in the late 1960s and 2001, which were not associated with substantial consumption declines. Comparing the panels, one area in which the models generate strong differences is persistence of the states. The i.i.d. model identifies recessions as a one-off negative shock, but since shocks are i.i.d., the agent does not forecast that the recession state will persist with high likelihood. In contrast, the two- and three-state models clearly show the persistence of the recession states. Disaster states are rare - after the initial transient post war period, there are only really two observations that place even modest probability on the disaster state - the recession in 1981 and the financial crisis at the end of 2008. This implies that disaster states are nearly 'Peso' events in the post WW2 sample.

The agent's beliefs are quite volatile early in the sample in all of the models. This is not surprising. Since initial parameter beliefs are highly uncertain, the agent has a very difficult time determining the current state of the economy as parameter uncertainty contributes to state uncertainty. As the agent learns, parameter uncertainty decreases and state identification is easier. It is important to note that even with full knowledge of the parameters, the agent will never be able to perfectly identify the state. ${ }^{18}$ The results also show that the priors do not have a large impact on the mean state beliefs, at least for the unrestricted two- and three-state models, as the posterior beliefs are roughly similar for the historical and look-ahead priors.

Next, consider beliefs over parameters. Due to the large number of parameters and in the interests of parsimony, we focus on a few of the more economically interesting and

\footnotetext{
${ }^{18}$ The posterior variance of the state, $\operatorname{var}\left[s_{t} \mid \mathcal{M}_{k}, y^{t}\right]$, does decline over time due to decreasing parameter uncertainty. This will be discussed further when we use GDP growth as an additional observation to help identify the state.
} 


\section{Figure 1 - Evolution of Mean State Beliefs}
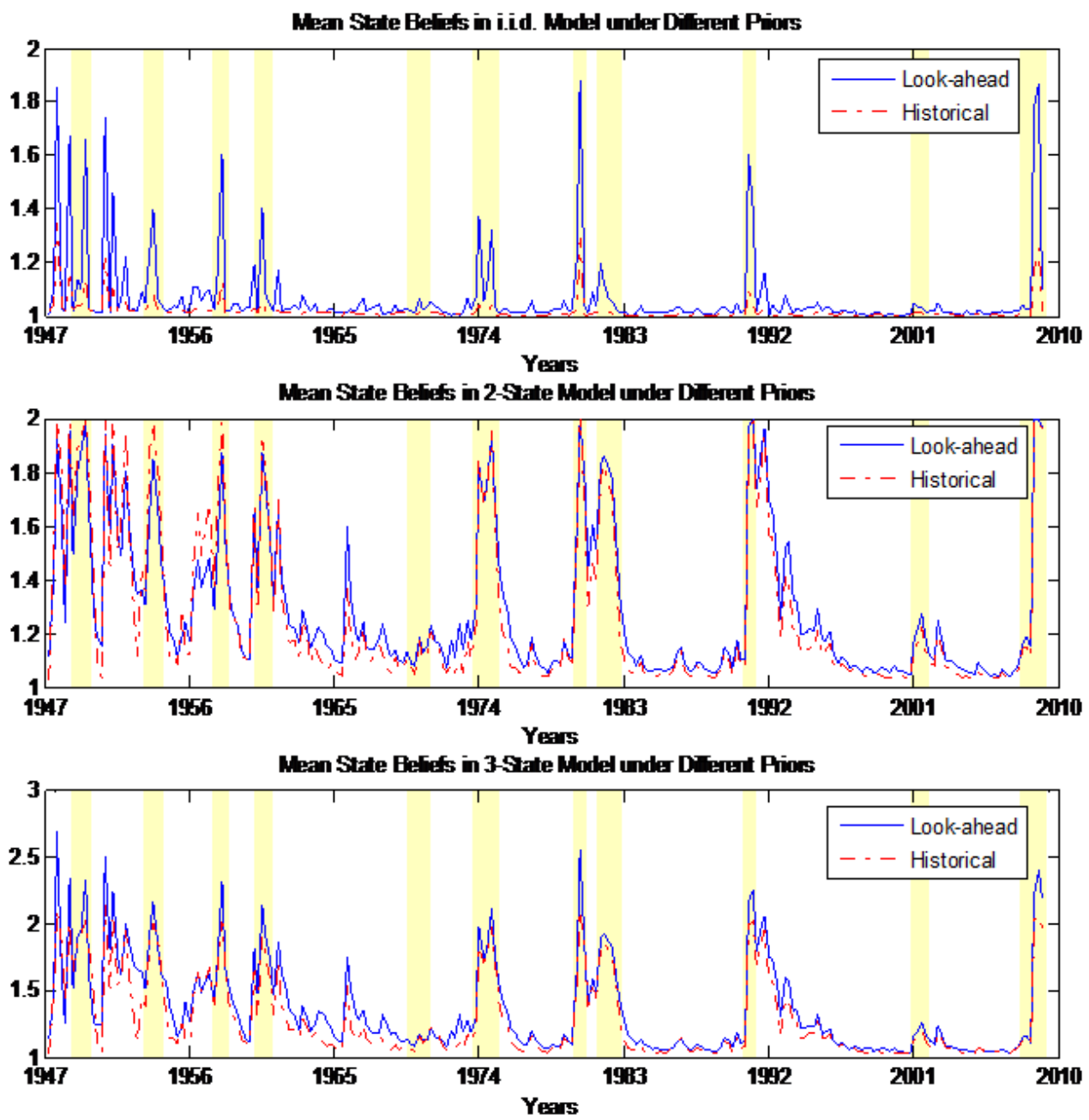

Figure 1: The plots show the means of agents' beliefs about the state of the economy at each point in time. " 1 " is an expansion good state, "2" is a recession state, and "3" is a disaster state. The models have either 2 or 3 states as indicated on each plot, and the time t state beliefs are formed using the history of consumption only up until and including time t. The "i.i.d. Model" is a model with i.i.d. consumption growth but that allows for jumps ("2" is a jump state). The sample is from 1947:Q2 until 2009:Q1. 
important parameters. For the two-state models, the top panels of Figure 2 display posterior means of the beliefs over $\sigma_{1}$ and $\sigma_{2}$, consumption growth volatility in the good and bad states, respectively. Notice that for the Historical prior the conditional volatilities slowly decrease, after a short (about 5 year) burn-in period, essentially throughout the sample. This is a combination of the Great Moderation (realized consumption volatility did decrease over the post-war sample) and the initial beliefs, which based on the historical experience expected higher consumption growth volatility. Interestingly, for the look-ahead prior, which is centered at the end of sample posterior values, the agents quickly unlearns the low full sample consumption growth volatility, and after about 5-year burn-in, the volatility is close to that observed for the historical prior. This occur because volatility was higher in the first portion of the sample. The subsequent decline in the volatility in the good state is quantitatively large (about a 30\% drop).

The lower panels in Figure 2 display the transition probabilities, $\pi_{11}$ and $\pi_{22}$. After the burn-in period, the first is essentially increasing over the sample, while the latter is decreasing. That is, 50 years of, on average, long expansions and high consumption growth leads to revisions in beliefs that are manifested in higher probabilities of staying in the good state and lower probabilities of staying in recession state. The probability of staying in a recession, conditional on being in a recession, goes down from about 0.85 to 0.75 . Clearly, such positive shocks to the agents' perception of the data generating process will lead to higher ex post equity returns than compared to ex ante expectations.

Figure 3 displays estimates of the mean parameters, $E\left[\mu_{i} \mid \mathcal{M}_{k}, y^{t}\right]$ for $i=1,2,3$ as well as a two-posterior standard deviation band for the 3 -state model using the historical prior. Learning is most apparent in the good state and least apparent in the disaster state. This is intuitive, since the economy spends most of its time in the good state and little, if any, time in the disaster state. This provides empirical evidence supporting the argument that a high level of parameter uncertainty is a likely feature of a model with a rarely observed state and is an important feature for disaster risk models (see also Chen, Joslin, and Tran, 2010). There is additional interesting time-variation in beliefs about the parameters, but this time-variation is best summarized via the total impact across all parameters, which is measured via predictive moments and discussed in the next section. 
Figure 2 - Evolution of Mean Parameter Beliefs
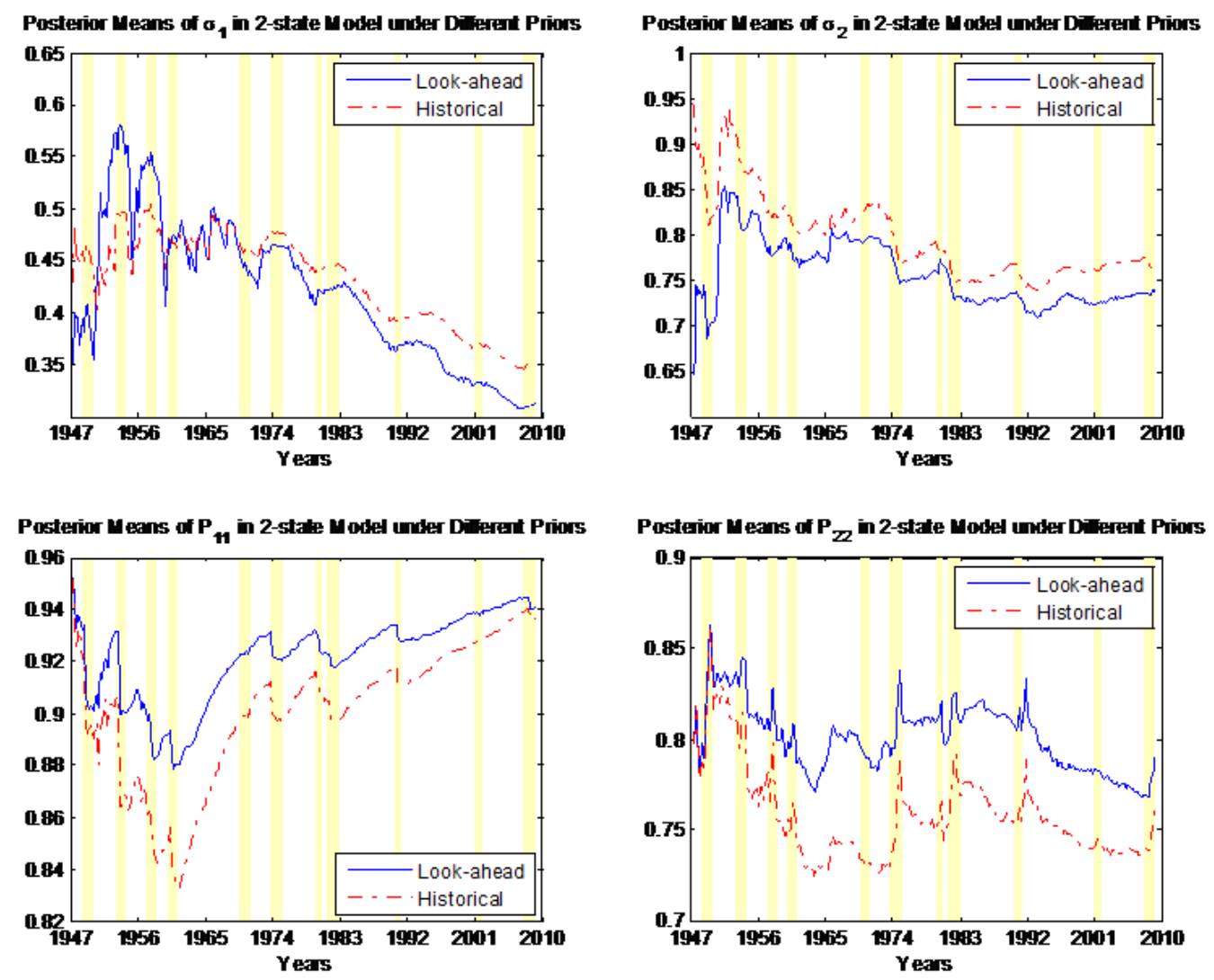

Figure 2: The two top plots in this figure show the mean beliefs of the volatility parameters within each state for the 2-state model, based on historical consumption data only. The two lower plots show the mean beliefs of the probabilities of remaining in the current state. The sample is from 1947:Q2 until 2009:Q1. 
Figure 3 - Evolution of Mean Parameter Beliefs

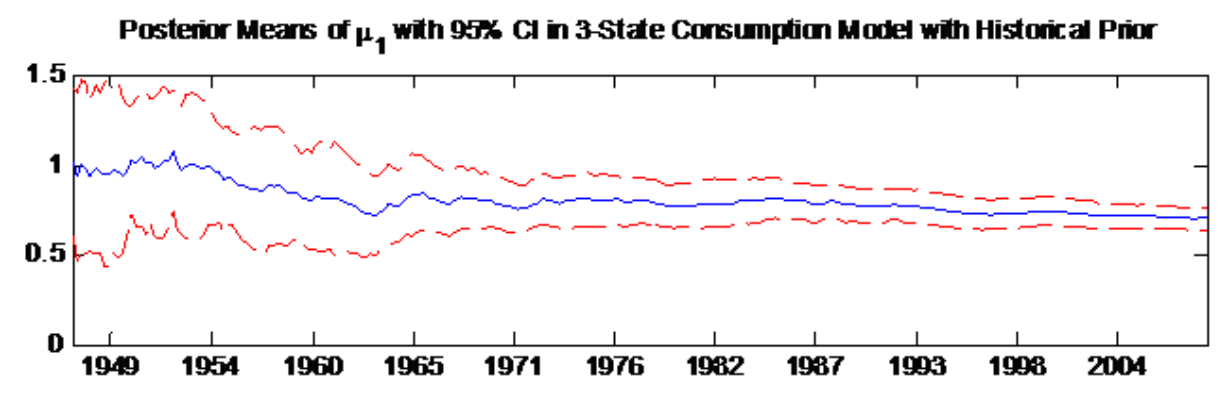

Posterior Mears of $\mu_{2}$ with $98 \% \mathrm{CI}$ in 3-State Consumption Moded with Historical Prior

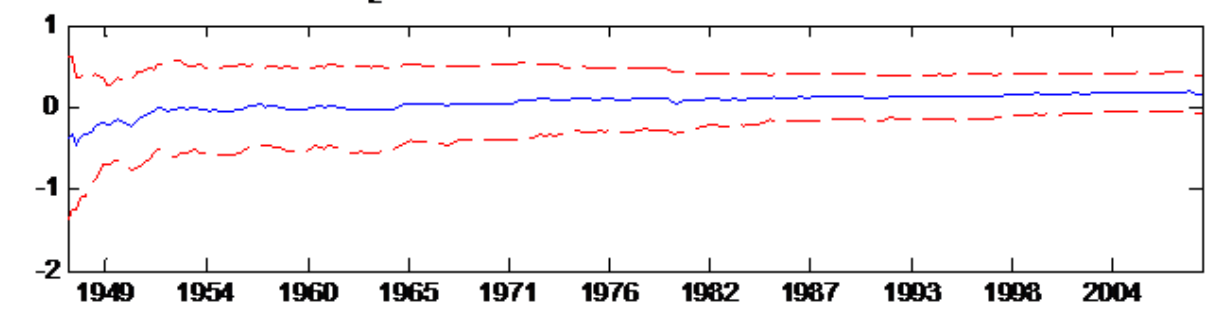

Posterior Mears of $\mu_{3}$ with $95 x$ C1 in 3-State Consumption Model with Historical Prior

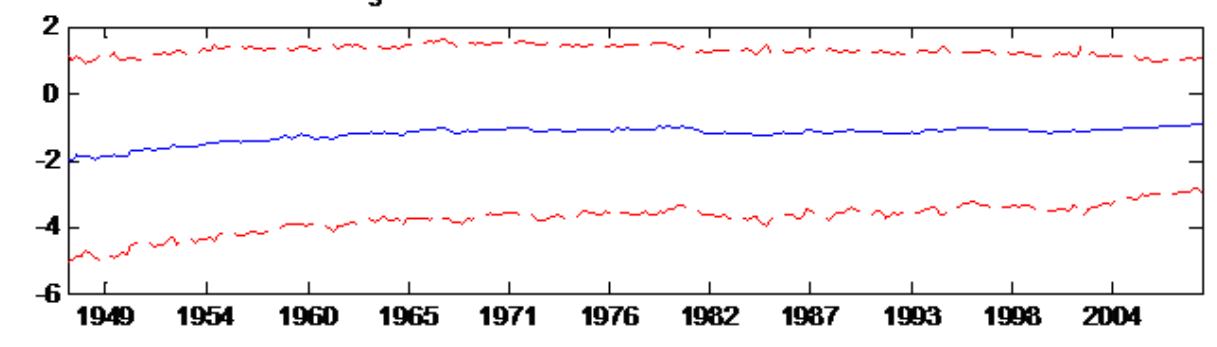

Figure 3: This figure shows the average and 2 standard deviation bounds of agents' time t beliefs about the mean parameters in the 3-state model, where only consumption data is used. The sample is from 1947:Q2 until 2009:Q1. 


\subsection{Beliefs about models and consumption dynamics}

The results of the previous section indicate that beliefs about the parameters vary through the sample, even for the look-ahead prior, but it is not clear from this what the overall net effect on quantities of interest such as the conditional volatility of consumption growth is. For example, if the probability of the bad state, which has higher consumption growth volatility, decreases, this could be offset by increase the consumption volatility in the good state. To provide asset-pricing relevant measures, we report the agent's beliefs regarding the first four moments of conditional consumption growth and model probabilities. All of these quantities are marginal, integrating out parameter, state, and/or model uncertainty. For example, the predictive mean for a given model, $\mathcal{M}_{k}$, is

$$
E\left[\Delta c_{t+1} \mid \mathcal{M}_{k}, y^{t}\right]=\int \Delta c_{t+1} p\left(\Delta c_{t+1} \mid \theta, s_{t}, \mathcal{M}_{k}, y^{t}\right) p\left(\theta, s_{t} \mid \mathcal{M}_{k}, y^{t}\right) d \theta d s_{t}
$$

which accounts for parameter and state uncertainty. In describing these moments, we generally abstract from the first ten years and treat it is a 'burn-in' period, in order to allow the prior some time to adjust to the data, as there is some transient volatility over these first few years.

The top panels in Figures 4 and 5 (for historical and look-ahead priors, respectively) display the conditional expected quarterly consumption growth for each model. Clearly, the two and three-state models generate relatively modest differences - both pick up business cycle fluctuations in expected consumption growth. Persistent recessions are missing from the i.i.d. model, as expected. All three models exhibit a modest low frequency increase in expected consumption growth over the first half of the sample. Recessions are associated, in all models, with lower expected consumption growth. The three-state models, which include a disaster state, do not feature a significantly lower expected consumption growth in recessions than the two-state models, as the realized recessions simply have not been severe enough to be clearly classified as disasters based on our priors.

The middle panels display marginal model probabilities, $p\left(\mathcal{M}_{k} \mid y^{t}\right)$. For simplicity, the prior probability of each model was set to $1 / 3$. Note first that the posterior probability of the i.i.d. model decreases relatively quickly towards zero. Thus, i.i.d. consumption growth is rejected by a Bayesian agent that updates by observing past realized consumption growth. Although not reported for brevity, this conclusion is robust even if the prior probability of the i.i.d. model is set to 0.95 - in this case it takes somewhat longer (a little over half the 


\section{Figure 4 - Quarterly Expected Consumption Growth}
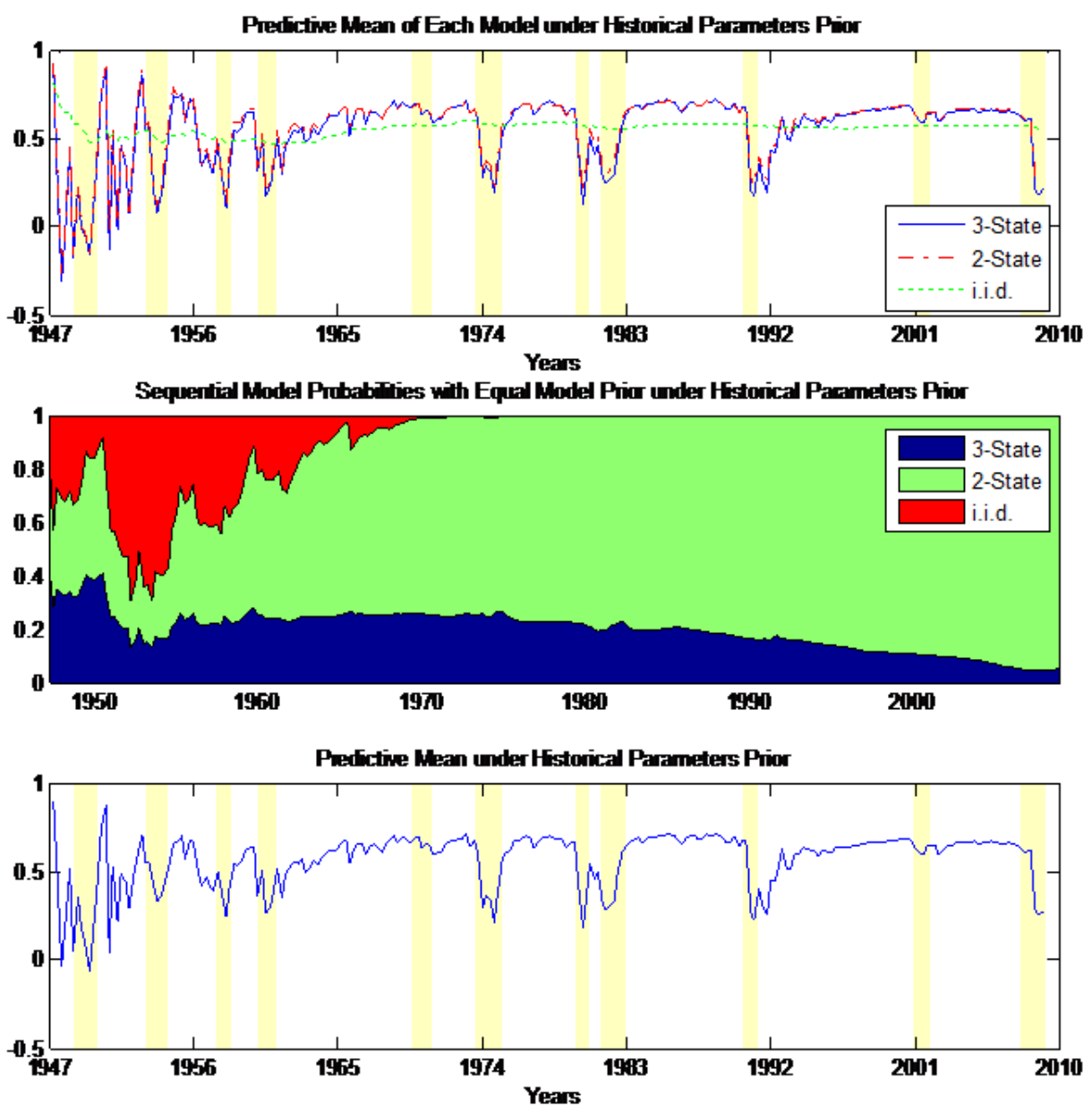

Figure 4: The top panel shows the quarterly conditional expected consumption growth, computed using the Historical Prior, from the three benchmark models: the "i.i.d. model", and hidden 2and 3-state switching regime models. The "i.i.d. Model" is a model with i.i.d. consumption growth but that allows for jumps. The middle plot shows the evolution of the probability of each model being the true model, where the prior at the beginning of the sample gives each model an equal probability. The lower plot shows the expected quarterly conditional consumption growth as perceived by an agent that also takes into account the model uncertainty (as given in the middle plot). The data in all cases go from 1947:Q2 - 2009:Q1. 


\section{Figure 5 - Quarterly Expected Consumption Growth}

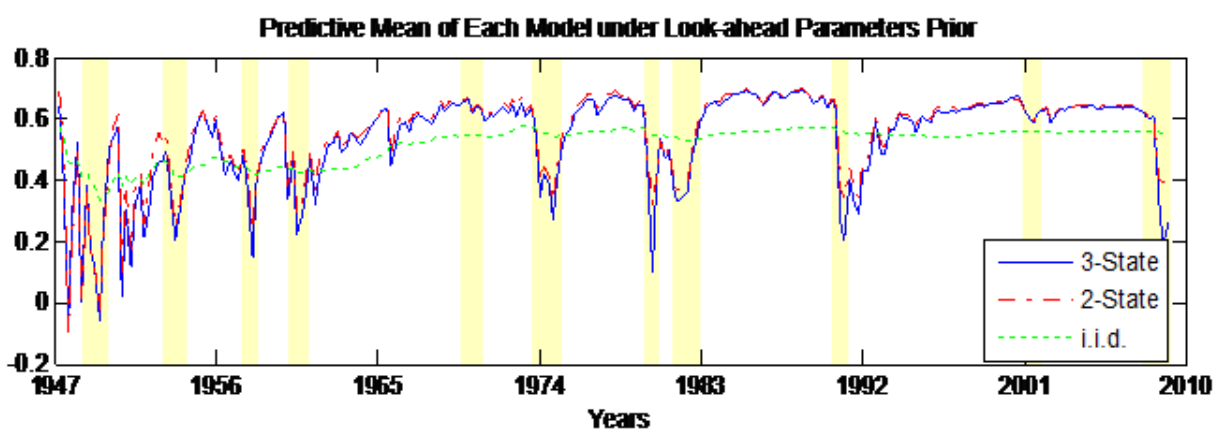

Sequential Moded Pobabinies with Equal Moded Prior under Look ahead Parameters Pior

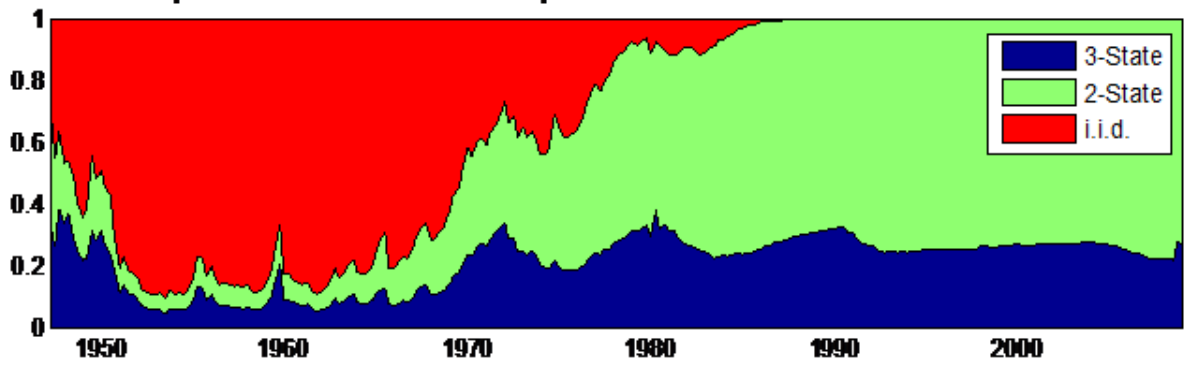

Predictive Mean under Lonk-ahead Parameters Phior

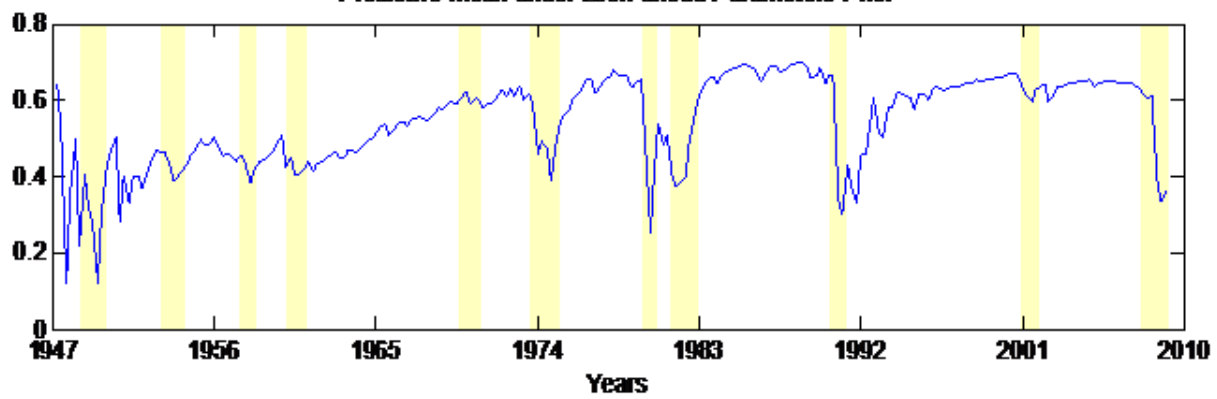

Figure 5: The top panel shows the quarterly conditional expected consumption growth, computed using the Rational Expectations Prior, from the three benchmark models: the "i.i.d. model", and hidden 2- and 3-state switching regime models. The "i.i.d. Model" is a model with i.i.d. consumption growth but that allows for jumps. The middle plot shows the evolution of the probability of each model being the true model, where the prior at the beginning of the sample gives each model an equal probability. The lower plot shows the expected quarterly conditional consumption growth as perceived by an agent that also takes into account the model uncertainty (as given in the middle plot). The data in all cases go from 1947:Q2 - 2009:Q1. 
sample), but the probability still drops very close to zero for the i.i.d. case. ${ }^{19}$ The 3 -state model also sees a reduction in its likelihood and ends at a 5-10\% probability level at the end of the sample. As mentioned in the introduction, a single large negative consumption shock would quickly change these probabilities. Thus, there are large changes in the model uncertainty across the sample.

The fact that agent can learn that consumption growth is not i.i.d. is important. Many asset pricing models specify i.i.d. consumption growth with the implicit assumption that it is not possible or difficult to detect non-i.i.d. dynamics in consumption. Our results show that agents, using only consumption growth data, can detect non-i.i.d. dynamics, and, can do so in real time, which is an even stronger result. The agent does not need to wait until the end of the sample. The results holds for various prior specifications. Although not pursued here, it would be interesting to see if Bayesian agents could detect in real-time non i.i.d. behavior in other models, such as Bansal and Yaron (2004).

The lower panels of Figures 4 and 5 show model averaged expected quarterly consumption growth. Model uncertainty does not have a strong effect on the expected consumption growth series in the 2- or 3-state models, as the conditional expected growth in each model is similar. This will be important for results below.

Turning to the conditional volatility of quarterly consumption growth, Figures 6 and 7 display a long-term downward trend in consumption growth volatility, with marked increases during recessions. The secular decline is largely driven by downward revisions in estimates of volatility as realized consumption growth was less volatile in the second half of this century. This is particularly strong for the historical prior, as the conditional volatility of consumption growth decreases from about $1 \%$ per quarter to about $0.5 \%$. Interestingly, the look-ahead prior has a similar trend, after a short burn-in period, though the size of the effect is about half as large. This is the Great Moderation - the fact that consumption volatility has decreased also over the post-war sample. In the models considered here, the decrease happens gradually, in contrast to studies that find ex post evidence of structural breaks or regime shifts at certain dates (e.g., Lettau, Ludvigson, and Wachter, 2008). Thus, the agent who learns in real time perceives that consumption volatility decreases gradually and not suddenly. This has important pricing implications as discussed later.

\footnotetext{
${ }^{19}$ Also, reported in the Appendix, this conclusion is robust to time-averaging of the consumption data: taking out an autocorrelation of 0.25 from the consumption growth data, which would be due to timeaveraging of i.i.d. data (see Working (1960)), does not qualitatively change these results - if anything it makes the rejection of the i.i.d. model occur sooner.
} 


\section{Figure 6 - Quarterly Consumption Growth Standard Deviation}
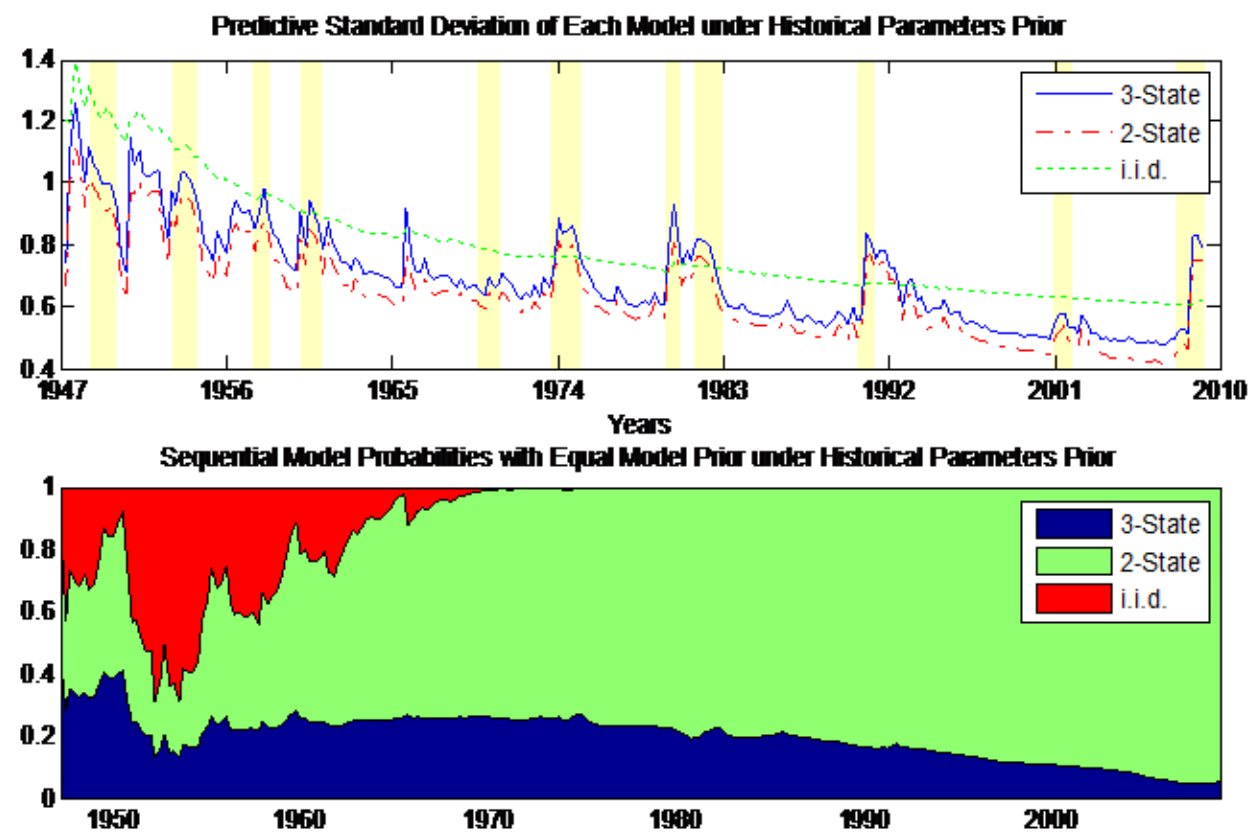

Predictie Standand Deviation under Fistoical Parameters Piir

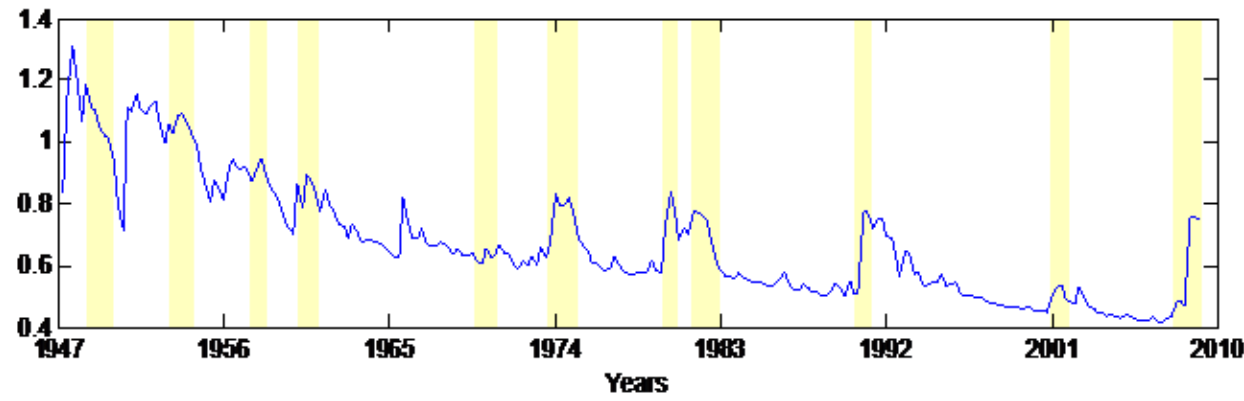

Figure 6: The top panel shows the quarterly conditional standard deviation of consumption growth, computed using the Historical Prior, from the three benchmark models: the "i.i.d. model", and hidden 2- and 3-state switching regime models. The "i.i.d. Model" is a model with i.i.d. consumption growth but that allows for jumps. The middle plot shows the evolution of the probability of each model being the true model, where the prior at the beginning of the sample gives each model an equal probability. The lower plot shows the quarterly conditional standard deviation of consumption growth, as perceived by an agent that also takes into account the model uncertainty (as given in the middle plot). The data in all cases go from 1947:Q2 - 2009:Q1. 
Figure 7 - Quarterly Consumption Growth Standard Deviation

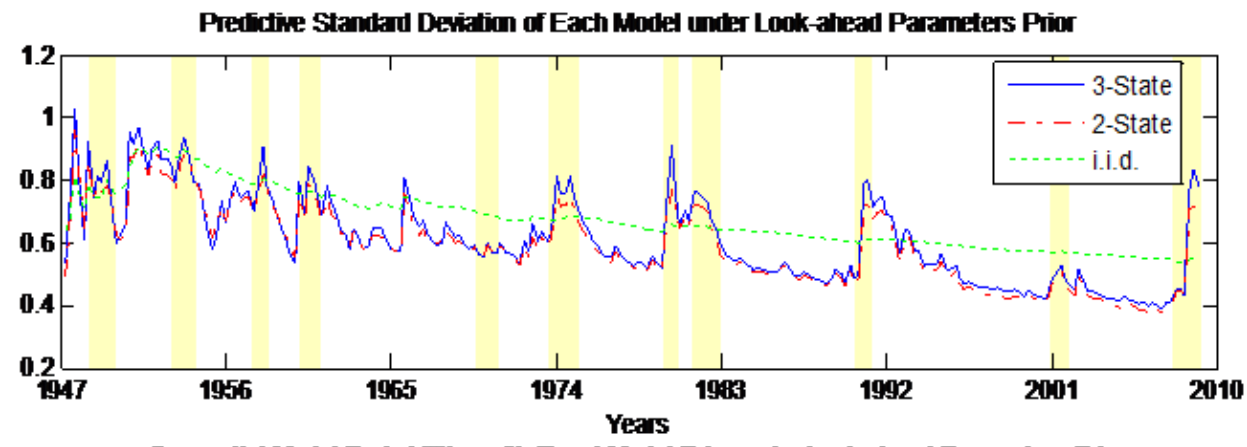

Sequential Moded Pubabities with Equal Moded Prior under Look ahead Parameters Pior

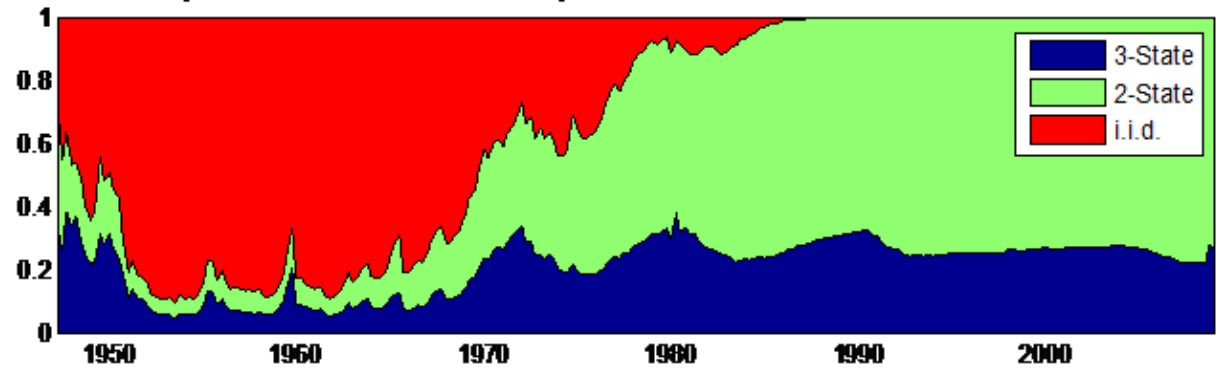

Predictive Standaud Devialion under Lonkahead Parameters Pīor

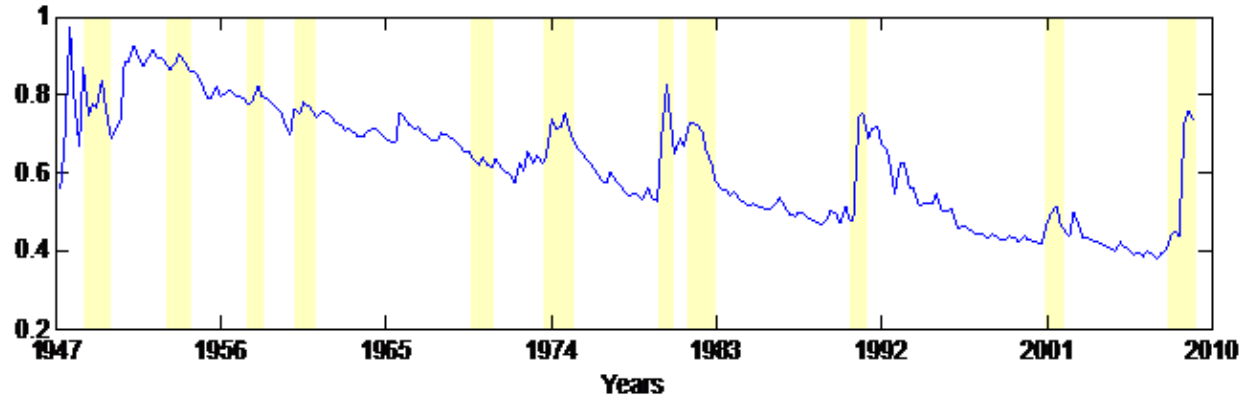

Figure 7: The top panel shows the quarterly conditional standard deviation of consumption growth, computed using the Rational Expectations Prior, from the three benchmark models: the "i.i.d. model", and hidden 2- and 3-state switching regime models. The "i.i.d. Model" is a model with i.i.d. consumption growth but that allows for jumps. The middle plot shows the evolution of the probability of each model being the true model, where the prior at the beginning of the sample gives each model an equal probability. The lower plot shows the quarterly conditional standard deviation of consumption growth, as perceived by an agent that also takes into account the model uncertainty (as given in the middle plot). The data in all cases go from 1947:Q2 - 2009:Q1. 
Every recession is associated with higher consumption growth volatility, although the size of the increase varies. The largest increase, on a percentage basis, occurs with the financial crisis of 2008. The increase is largest in the three-state model, as the mean state belief at this time approaches the third state, which has a very high volatility. There is little updating about the volatility of the disaster state through the sample, since there have been no prolonged visits to this state. Thus, this reflects the fear that prevailed in the fall of 2008 that the economy was potentially headed into a depression not seen since the 1930s. This econometric result squares nicely with anecdotes from the crisis.

The middle plots of Figures 6 and 7 show the model probabilities through the sample. Model probabilities could be driven by unexpected volatility, but this does not appear to be a primary determinant. The lower plot again shows that conditional consumption growth volatility is not particularly affected by model uncertainty, since both the two and the threestate models have similar volatility patterns, and since the i.i.d. model is essentially phased out in the first half of the sample.

Figures 8 and 9 show the time-variation in expected consumption skewness and kurtosis, respectively, for the historical prior, again including the model probabilities and model averaged estimates. The results are similar for the look-ahead prior (after a burn-in period) and are not shown. The time-variation in the conditional skewness is dominated by business cycle variation for the two and three-state models, and there is not a clear low frequency trend. When the economy is in a recession, consumption growth is naturally less negatively skewed for two reasons: (1) there is a high probability that the economy jumps to a higher (i.e. better) state and (2) expected consumption volatility is high, which tends to decrease skewness.

Conditional kurtosis is lower in bad states as these states are the least persistent and volatility is highest. Large, rare, outcomes are more likely when the economy is in the good state. This has potentially interesting option pricing implications (see, e.g., Backus, Chernov, and Martin (2009)), as the skewness and kurtosis will be related to volatility smiles. It is worth noting that parameter uncertainty gives an extra kick to conditional skewness and kurtosis measures relative to the case of fixed parameters, where the skewness and kurtosis both move little over time (the fixed parameter case is not reported here for brevity). Unlike the first two moments, there are now relatively large differences between the two and threestate models. The three-state model has significantly more negative conditional skewness and higher conditional kurtosis than the three-state model, both due to the presence of the 


\section{Figure 8 - Quarterly Consumption Growth Skewness}
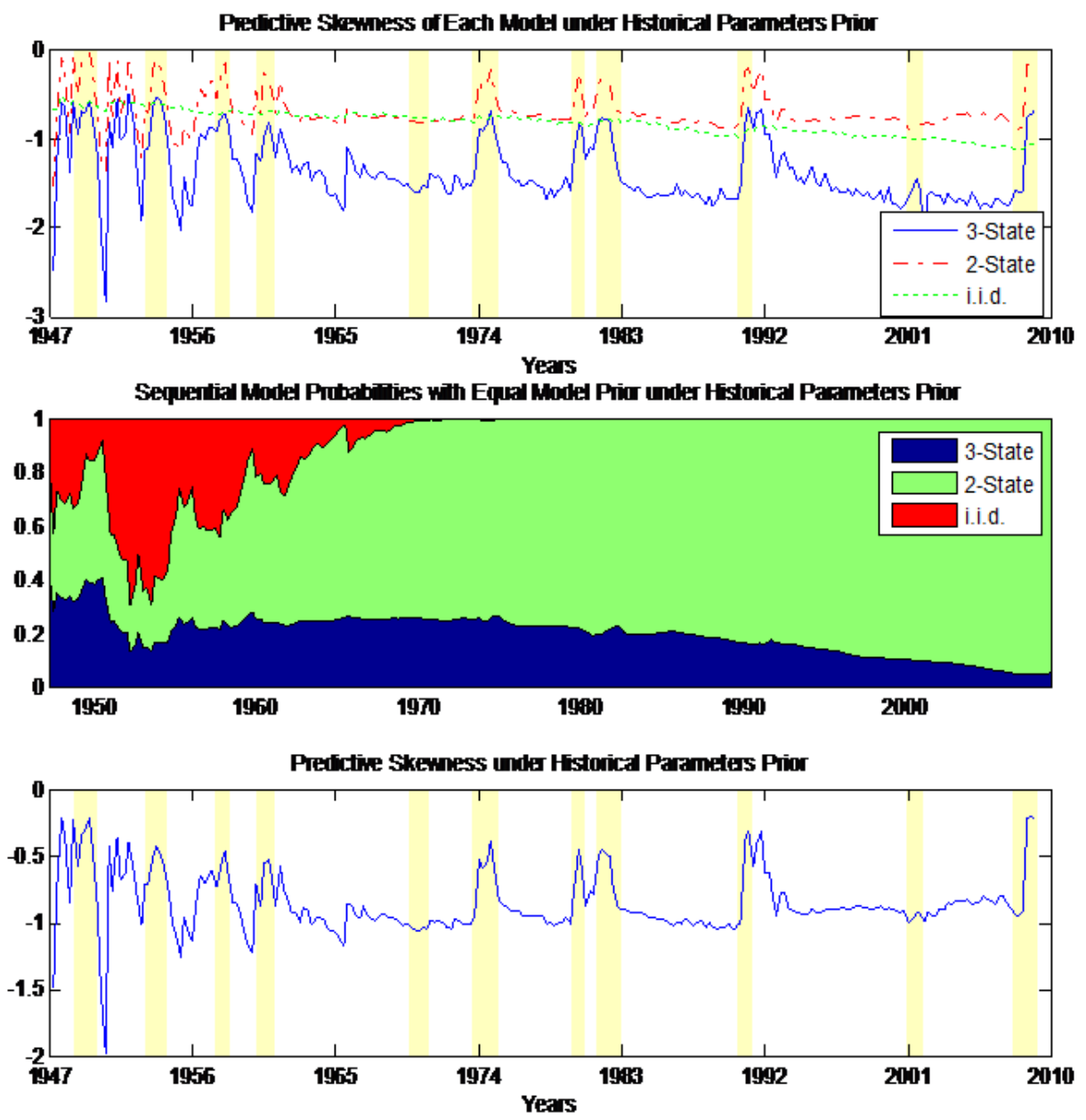

Figure 8: The top panel shows the quarterly conditional skewness of consumption growth, computed using the Historical Prior, from the three benchmark models: the "i.i.d. model", and hidden 2and 3-state switching regime models. The "i.i.d. Model" is a model with i.i.d. consumption growth but that allows for jumps. The middle plot shows the evolution of the probability of each model being the true model, where the prior at the beginning of the sample gives each model an equal probability. The lower plot shows the quarterly conditional skewness of consumption growth, as perceived by an agent that also takes into account the model uncertainty (as given in the middle plot). The data in all cases go from 1947:Q2 - 2009:Q1. 
disaster-state. Interestingly, the differences are greater in expansions than in recessions.

Because of differences between the two and three-state models, the model averaged measures of skewness and kurtosis are more interesting. In particular, the three-state model, with its disaster state, has very high negative skewness and very high excess kurtosis, so even though the probability of this model being the right model goes quite low, it still impacts consumption moments after model uncertainty is integrated out. Thus, among the models considered here, model uncertainty and its dynamic behavior is likely to have the strongest implications for assets such as out-of-the-money options that are more sensitive to the tail behavior of consumption growth.

\subsection{Does learning matter for asset prices?}

The previous results indicate that the agent's beliefs - about parameters, moments, and models - vary substantially over time and across the business cycle. If learning is an important determinant of asset prices, changes in beliefs should be a significant determinant of asset returns. This is a fundamental test of the importance of learning about the consumption dynamics. For example, if agents learn that expected consumption growth is higher than previously thought, this revision in beliefs will be reflected in the aggregate wealthconsumption ratio (if the elasticity of intertemporal substitution is different from one). In particular, if the substitution effect dominates, the wealth-consumption ratio will increase when agents revise their beliefs about the expected consumption growth rate upwards (see, e.g., Bansal and Yaron (2004)). As another example, if agents learn that aggregate risk (consumption growth volatility) is lower than previously thought, this will generally lead to a change in asset prices as both the risk premium and the risk-free rate are affected. In the Bansal and Yaron (2004) model, an increase in the aggregate volatility leads to a decrease in the stock market's price-dividend ratio.

To test this, we regress excess quarterly stock returns (obtained from Kenneth French's website) on changes in beliefs about expected consumption growth and expected consumption growth volatility. We use the beginning of period timing for the consumption data here and elsewhere in the paper. ${ }^{20}$ The regressors are the shocks, $E_{t}\left(\Delta c_{t+1}\right)-E_{t-1}\left(\Delta c_{t+1}\right)$ and

\footnotetext{
${ }^{20}$ Due to time-averaging (see Working, 1960), Campbell (1999) notes that one can use either beginning of period or end of period consumption in a given quarter as the consumption for that quarter. The beginning of period timing yields stronger results than using the end of period convention (although the signs are the same in the regressions). In principle, the results should be the same, so this is consistent with some
} 


\section{Figure 9 - Quarterly Consumption Growth Kurtosis}
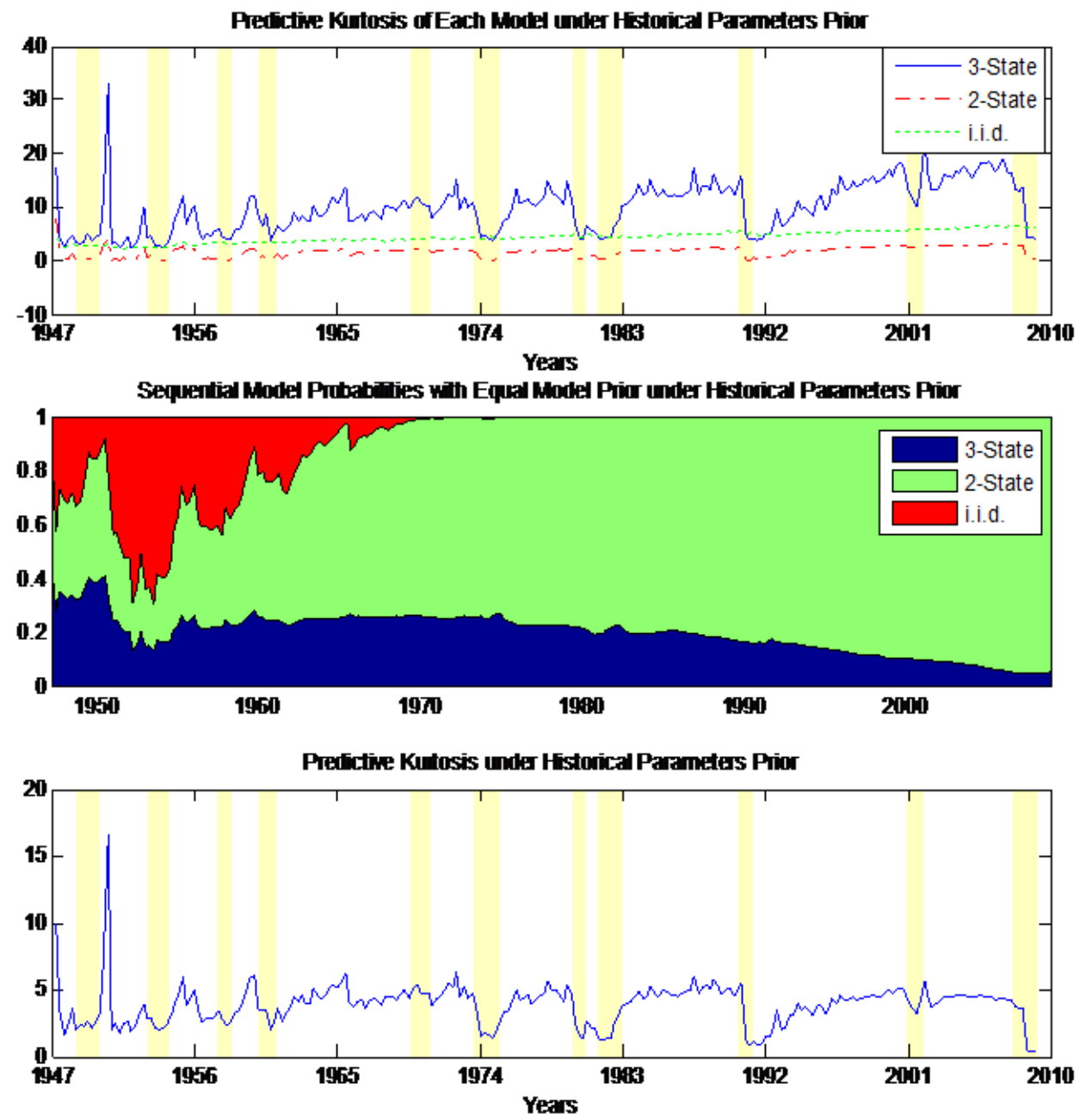

Figure 9: The top panel shows the quarterly conditional excess kurtosis of consumption growth, computed using the Historical Prior, from the three benchmark models: the "i.i.d. model", and hidden 2- and 3-state switching regime models. The "i.i.d. Model" is a model with i.i.d. consumption growth but that allows for jumps. The middle plot shows the evolution of the probability of each model being the true model, where the prior at the beginning of the sample gives each model an equal probability. The lower plot shows the quarterly conditional excess kurtosis of consumption growth, as perceived by an agent that also takes into account the model uncertainty (as given in the middle plot). The data in all cases go from 1947:Q2 - 2009:Q1. 
$\sigma_{t}\left(\Delta c_{t+1}\right)-\sigma_{t-1}\left(\Delta c_{t+1}\right)$. Notice that the only thing that is changing is the conditioning information set as we go from time $t-1$ to time $t$; the regressors are revisions in beliefs. We calculate these conditional moments for each prior integrating out state, model and parameter uncertainty. The first 10 years of the sample are used as a burn-in period to remove the high volatility and alleviate any prior misspecification, and these observations are therefore not included in the regressions.

Separate regressions are run for the historical and look-ahead priors, and we control for contemporaneous consumption growth and lagged consumption growth (the direct cash flow effect). By controlling for realized consumption growth, we ensure that the results are driven by model-based revisions in beliefs, and not just the fact that realized consumption growth (a direct cash flow effect) was, for example, unexpectedly high. To separate out the effects of parameter from state learning, we use revisions in expected consumption growth beliefs computed from the three-state model with fixed parameters (set to their full-sample values) as an additional control. ${ }^{21}$

Specifications 1 and 2 in Panel A (historical prior) and Panel B (look-ahead prior) in Table 1 show that increases in expected conditional consumption growth are positively and strongly significantly associated with excess contemporaneous stock returns for both priors. This result holds controlling for contemporaneous and lagged consumption growth (the direct cash flow effect), and so we can equivalently say that revisions in beliefs are significantly related to shocks to the price-dividend ratio. This is a very strong result, pointing to the importance of a learning-based explanation for realized stock returns. These results could be driven by parameter or state learning.

Specification 3 shows the results are robust to the inclusion of the update in expected consumption growth that arise in a model with fixed parameters. That is, updates in expectations when learning about about parameters, states, and models are significantly related to contemporaneous stock returns, while the corresponding updates in expectations based on a single model with known parameters but hidden states estimated on the full sample - the standard practice in the literature - are not. This result is mostly driven by parameter learning - the previous section showed the expected consumption growth estimates are quite similar for different model specifications. In other words, parameter learning is important for understanding the time-series of the market valuation ratio in the post-WW2 U.S. data. To

information being impounded in stocks before the consumption data is revealed to the Bureau of Economic Analysis.

${ }^{21}$ Using the fixed parameter 2-state model as the control instead does not change the results. 
our knowledge, this is the first direct comparison of learning about models and parameters versus the traditional implementation of the rational expectation explanations based on the actual sequence of realized shocks.

For the variance (regression specifications 4 and 5 in Table 1) we get the opposite result, as one would expect (at least with a high elasticity of intertemporal substitution, as we will use later in the paper): unexpected increases in conditional consumption growth variance are associated with negative contemporaneous stock returns. This result is not significant at the $5 \%$ level when including contemporaneous and lagged consumption growth in the regressions (specification 5). This does not mean there is no effect, we just cannot distinguish it from the direct cash flow effect when learning from consumption data alone.

To summarize, we find strong evidence that the updates in beliefs elicited from our model/prior combinations are associated with actual updates in agent beliefs at the time, as proxied by stock market returns. Again, it is important to recall that no asset price data was used to generate these belief revisions.

\subsection{Learning from additional macro variables}

Agents have access to more than just aggregate consumption growth data when forming beliefs. Here we provide one approach for incorporating this additional information and apply this methodology to learning from quarterly GDP growth, in addition to consumption. Suppose $x_{t}$ represents the common growth factor in the economy and evolves via:

$$
x_{t}=\mu_{s_{t}}+\sigma_{s_{t}} \varepsilon_{t}
$$

where $\varepsilon_{t} \stackrel{i . i . d .}{\sim} \mathcal{N}(0,1)$, and $s_{t}$ is the state of the economy, which follows the same Markov chains specified earlier. Consumption growth $\Delta c$ and $J$ additional variables $Y_{t}=\left[y_{t}^{1}, y_{t}^{2}, \ldots, y_{t}^{J}\right]^{\prime}$ are assumed to follow:

$$
\begin{gathered}
\Delta c_{t}=x_{t}+\sigma_{c} \varepsilon_{t}^{c}, \\
y_{t}^{j}=\alpha_{j}+\beta_{j} x_{t}+\sigma_{j} \varepsilon_{t}^{j}, \text { for } j=1,2, \ldots, J
\end{gathered}
$$

where $\varepsilon_{t}^{c} \stackrel{i . i . d .}{\sim} \mathcal{N}(0,1)$, and $\varepsilon_{t}^{j} \stackrel{i . i . d .}{\sim} \mathcal{N}(0,1)$ for any $j$. Note that the coefficients in equation (5) are not state dependent, which implies that the additional variables will primarily aid in state identification. The specification allows for the additional observation variables to be 


\section{Table 1 - Updates in Beliefs versus Realized Stock Returns}

Table 1: The table shows the results from regressions of innovations in agents' expectations of future consumption growth $\left(E_{t+1}\left[\Delta c_{t+2}\right]-E_{t}\left[\Delta c_{t+2}\right]\right)$ and conditional consumption growth variance $\left(\sigma_{t+1}^{2}\left[\Delta c_{t+2}\right]-\sigma_{t}^{2}\left[\Delta c_{t+2}\right]\right)$ versus contemporaneous excess stock market returns. In calculating the expectations, the parameter and model uncertainty is integrated out. The controls are lagged and contemporaneous realized log consumption growth, as well as the innovation in expected consumption growth derived from the 3-state model with fixed parameters (i.e., no model or parameter uncertainty). Panel A shows the results for the Historical priors, while Panel B shows the results for the Look-ahead priors. Heteroskedasticity and autocorrelation adjusted (Newey-West; 3 lags) standard errors are used. ${ }^{*}$ denotes significance at the $10 \%$ level, ${ }^{* *}$ denotes significance at the $5 \%$ level, and ${ }^{* * *}$ denotes significance at the 1\% level. The sample is from 1947:Q2 until 2009:Q1. In the below regressions, we have removed the first 40 observations (10 years), as a burn-in period to alleviate misspecification of the priors. However, similar results are obtained with no burn-in or with 80 quarter burn-in.

\begin{tabular}{|c|c|c|c|c|c|}
\hline \multicolumn{6}{|c|}{ Dependent variable: $r_{m, t+1}-r_{f, t+1}$ (excess market returns) } \\
\hline Panel A: Historical prior & 1 & 2 & 3 & 4 & 5 \\
\hline$E_{t+1}\left[\Delta c_{t+2}\right]-E_{t}\left[\Delta c_{t+2}\right]$ & $\begin{array}{c}33.34^{* * *} \\
(8.60)\end{array}$ & $\begin{array}{c}22.79^{* *} \\
(11.27)\end{array}$ & $\begin{array}{l}44.26^{* *} \\
(20.18)\end{array}$ & & \\
\hline$\sigma_{t+1}^{2}\left[\Delta c_{t+2}\right]-\sigma_{t}^{2}\left[\Delta c_{t+2}\right]$ & & & & $\begin{array}{c}-30.38^{* * *} \\
(10.53)\end{array}$ & $\begin{array}{c}-10.96 \\
(9.50)\end{array}$ \\
\hline \multicolumn{6}{|l|}{ Controls: } \\
\hline$\Delta c_{t+1}$ & & $\begin{array}{c}1.74 \\
(1.66)\end{array}$ & & & $\begin{array}{c}3.77^{* * *} \\
(1.36)\end{array}$ \\
\hline ( & & $\begin{array}{l}2.54^{*} \\
(1.43)\end{array}$ & & & $\begin{array}{c}2.13 \\
(1.43)\end{array}$ \\
\hline$\left[E_{t+1}\left[\Delta c_{t+2}\right]-E_{t}\left[\Delta c_{t+2}\right]\right]_{\text {Fixed parameters }}^{3-\text { state model }}$ & & & $\begin{array}{l}-10.80 \\
(15.02)\end{array}$ & & \\
\hline$R_{a d j}^{2}$ & $8.6 \%$ & $10.9 \%$ & $8.3 \%$ & $4.7 \%$ & $9.6 \%$ \\
\hline Panel B: Look-ahead prior & 1 & 2 & 3 & 4 & 5 \\
\hline$E_{t+1}\left[\Delta c_{t+2}\right]-E_{t}\left[\Delta c_{t+2}\right]$ & $\begin{array}{c}54.41^{* * *} \\
(11.51)\end{array}$ & $\begin{array}{c}34.33^{* * *} \\
(12.21)\end{array}$ & $\begin{array}{c}52.39^{* *} \\
(21.84)\end{array}$ & & \\
\hline$\sigma_{t+1}^{2}\left[\Delta c_{t+2}\right]-\sigma_{t}^{2}\left[\Delta c_{t+2}\right]$ & & & & $\begin{array}{c}-41.06^{* * *} \\
(11.11)\end{array}$ & $\begin{array}{r}-20.14^{*} \\
(11.10)\end{array}$ \\
\hline \multicolumn{6}{|l|}{ Controls: } \\
\hline$\Delta c_{t+1}$ & & $\begin{array}{l}2.30^{*} \\
(1.43)\end{array}$ & & & $\begin{array}{l}3.53^{* *} \\
(1.36)\end{array}$ \\
\hline er & & $\begin{array}{l}2.31^{*} \\
(1.40)\end{array}$ & & & $\begin{array}{c}2.15 \\
(1.40)\end{array}$ \\
\hline$\left[E_{t+1}\left[\Delta c_{t+2}\right]-E_{t}\left[\Delta c_{t+2}\right]\right]_{\text {Fixed parameters }}^{3-\text { state model }}$ & & & $\begin{array}{c}1.32 \\
(11.86)\end{array}$ & & \\
\hline$R_{a d j}^{2}$ & $8.4 \%$ & $11.0 \%$ & $8.0 \%$ & $5.2 \%$ & $10.1 \%$ \\
\hline
\end{tabular}


stronger or weaker signals of the underlying state of the economy than consumption growth. For the case of GDP growth, this captures the idea that investments is more cyclical than consumption, which makes GDP growth a better business cycle indicator. The linearity of the relationship is an assumption that is needed for conjugate priors.

The similar conjugate priors for the parameters are applied. For each state $s_{t}=i$, $p\left(\mu_{i} \mid \sigma_{i}^{2}\right) p\left(\sigma_{i}^{2}\right) \sim \mathcal{N} \mathcal{I} \mathcal{G}\left(a_{i}, A_{i}, b_{i}, B_{i}\right)$, where $\mathcal{N} \mathcal{I} \mathcal{G}$ is the normal/inverse gamma distribution. $\sigma_{c}$ is assumed to follow an inverse gamma distribution $\mathcal{I} \mathcal{G}\left(b_{c}, B_{c}\right)$, and for each $j=1,2, \ldots, J$, $p\left(\left[\alpha_{j}, \beta_{j}\right]^{\prime} \mid \sigma_{j}^{2}\right) p\left(\sigma_{j}^{2}\right) \sim \mathcal{N} \mathcal{I} \mathcal{G}\left(a_{j}, A_{j}, b_{j}, B_{j}\right)$, where $p\left(\left[\alpha_{j}, \beta_{j}\right]^{\prime} \mid \sigma_{j}^{2}\right)$ is a bivariate normal distribution $\mathcal{N}\left(a_{j}, A_{j} \sigma_{j}^{2}\right), a_{j}$ is a $2 \times 1$ vector and $A_{j}$ is a $2 \times 2$ matrix. Particle filtering is straightforward to implement in this specification by modifying the algorithm described in the Appendix.

To analyze the implications of additional information, we consider learning using real, per capita U.S. GDP growth as an additional source of information. This exercise generates a battery of results: time series of parameter beliefs, conditional moments, and model probabilities. We report only a few interesting statistics in the interests of parsimony. Figure 10 shows that the state beliefs do not change dramatically, although GDP growth is typically thought of as more informative about business cycle fluctuations than consumption growth. To characterize how the additional data aids in state identification, we compute posterior standard deviations for the states, std $\left[s_{t} \mid \mathcal{M}_{k}, y^{t}\right]$, again integrating out parameter uncertainty. Figure 11, shows that indeed the uncertainty about the state is much lower (about half) than what was the case when using consumption growth as the only source of information. Thus, adding GDP growth to the agent's information set increases the precision of the state identification. ${ }^{22}$

The increased certainty about the state improves parameter identification also, which is confirmed in Figure 12. Here the uncertainty about the good and bad states mean consumption growth rates is lower, after a 10-year burn-in, than in the case using consumption as the only source of information. Adding GDP growth also results in a greater difference in expected consumption growth across the states. Figure 13 and 14 shows that the difference in the expected consumption growth rate in recessions versus expansions is about $0.6 \%$ per quarter, versus about $0.4 \%$ in the case of consumption information only (see Figure 4 and 5 ). The dynamic behavior of the conditional standard deviation of consumption growth is not

\footnotetext{
${ }^{22}$ It is technically feasible to impose cointegration between consumption and GDP by including the log consumption to GDP ratio on the right hand side of Equation (5). We thank Lars Hansen for pointing this out.
} 


\section{Figure 10 - Mean State Beliefs (GDP)}
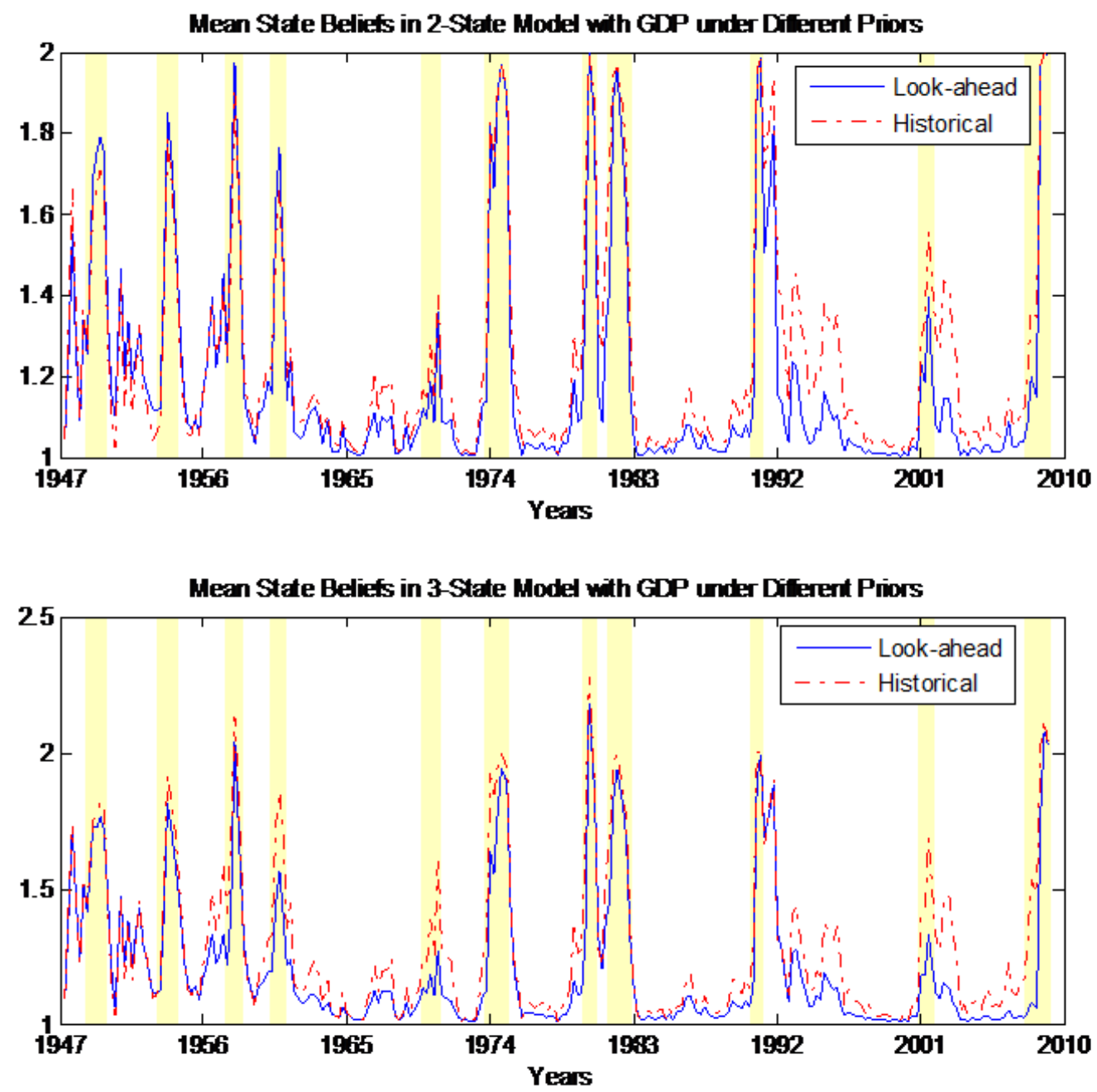

Figure 10: The figures show the means of agents' beliefs about the state of the economy at each point in time. "1" is an expansion good state, "2" is a recession state, and " 3 " is a disaster state. The models have either 2 or 3 states as indicated on each plot, and the time t state beliefs are formed using the history of both consumption and GDP up until and including time t. The sample is from 1947:Q2 until 2009:Q1. 
Figure 11 - Uncertainty about state (GDP)

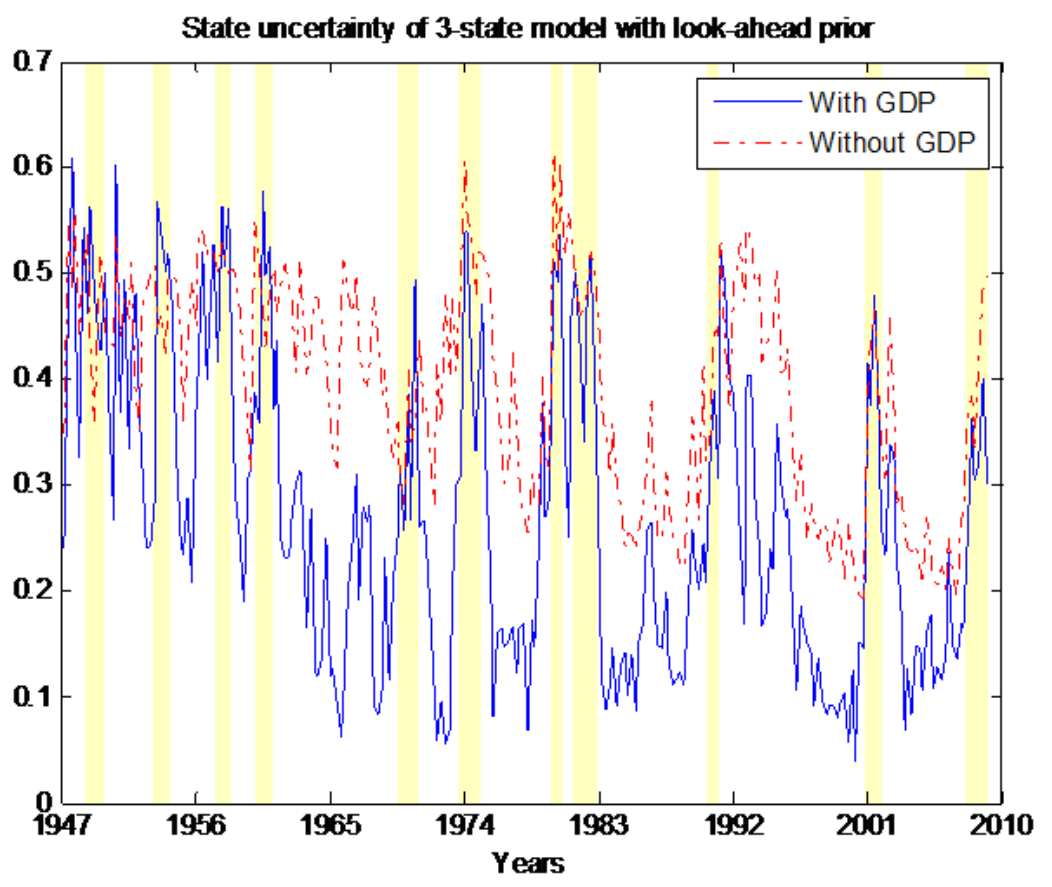

Figure 11: The graph shows the standard deviation of the posterior belief about the states for the case of Look-ahead priors when the consumption dynamics are estimated using consumption data only versus the consumption and GDP data. The sample is from 1947:Q2 until 2009:Q1. 
significantly changed (not reported for brevity). The model specification results are similar, as the data again favors the two-state model, leaving the three-state model with a very low probability at the end of the sample. It is noteworthy, however, that the probability of the three-state (disaster) model increases at the onset of the financial crisis in 2008. The results for the i.i.d. model are not reported here for brevity; its probability very quickly gets close to zero.

Table 2 shows the regressions of contemporaneous stock returns and updates in agent beliefs about conditional expected consumption growth and consumption growth variance, as calculated from this extended model. The results are similar, but in fact overall stronger than the results using only consumption growth. Updates in agent expectations about these moments are significantly related to stock returns, also after controlling for contemporaneous and lagged consumption growth and updates in expected consumption growth derived from a model with fixed parameters. Again, this is strong, preference-free evidence that learning about parameters and models is an important feature of the data.

\section{Asset pricing implications}

In this section, we evaluate the pricing implications of the models with parameter and model uncertainty. The model is solved at the quarterly frequency, and the representative agent is assumed to have Epstein and Zin (1989) preferences, which are defined recursively as:

$$
U_{t}=\left\{(1-\beta) C_{t}^{1-1 / \psi}+\beta\left(E_{t}\left[U_{t+1}^{1-\gamma}\right]\right)^{\frac{1-1 / \psi}{1-\gamma}}\right\}^{\frac{1}{1-1 / \psi}}
$$

where $C_{t}$ is the consumption, $\psi \neq 1$ is the intertemporal elasticity of substitution (IES) in consumption, and $\gamma \neq 1$ is the coefficient of relative risk aversion. These preferences imply the stochastic discount factor

$$
M_{t+1}=\beta\left(\frac{C_{t+1}}{C_{t}}\right)^{-\gamma}\left(\beta \frac{P C_{t+1}+1}{P C_{t}}\right)^{\frac{1 / \psi-\gamma}{1-1 / \psi}}
$$

where $P C_{t}$ is the wealth-consumption ratio - that is, the price-dividend ratio for the claim to the stream of future aggregate consumption. The first component of the pricing kernel is that which obtains under standard power utility, while the second component is present if 


\section{Figure 12 - Parameter uncertainty (GDP)}

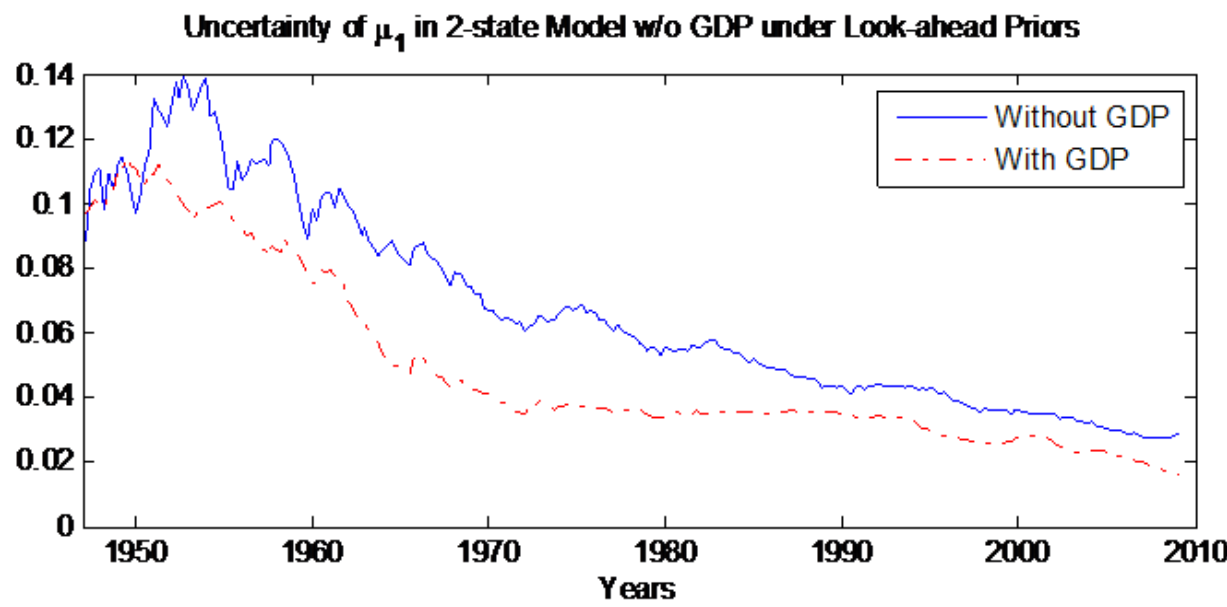

Uncertainty of $\mu_{2}$ in 2-state Model wo GDP under Look-ahead Priors

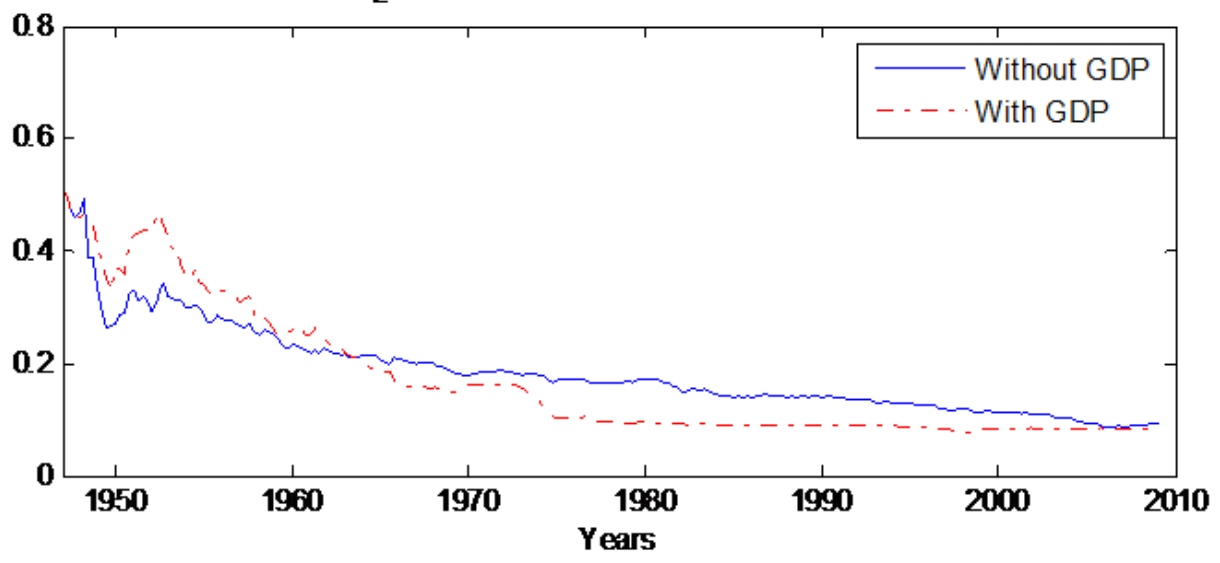

Figure 12: The graph shows the standard deviation of the posterior belief about some of the parameters for the case of Historical priors when the consumption dynamics are estimated using consumption data only versus the consumption and GDP data. The sample is from 1947:Q2 until 2009:Q1. 


\section{Figure 13 - Conditional expected consumption growth (GDP)}
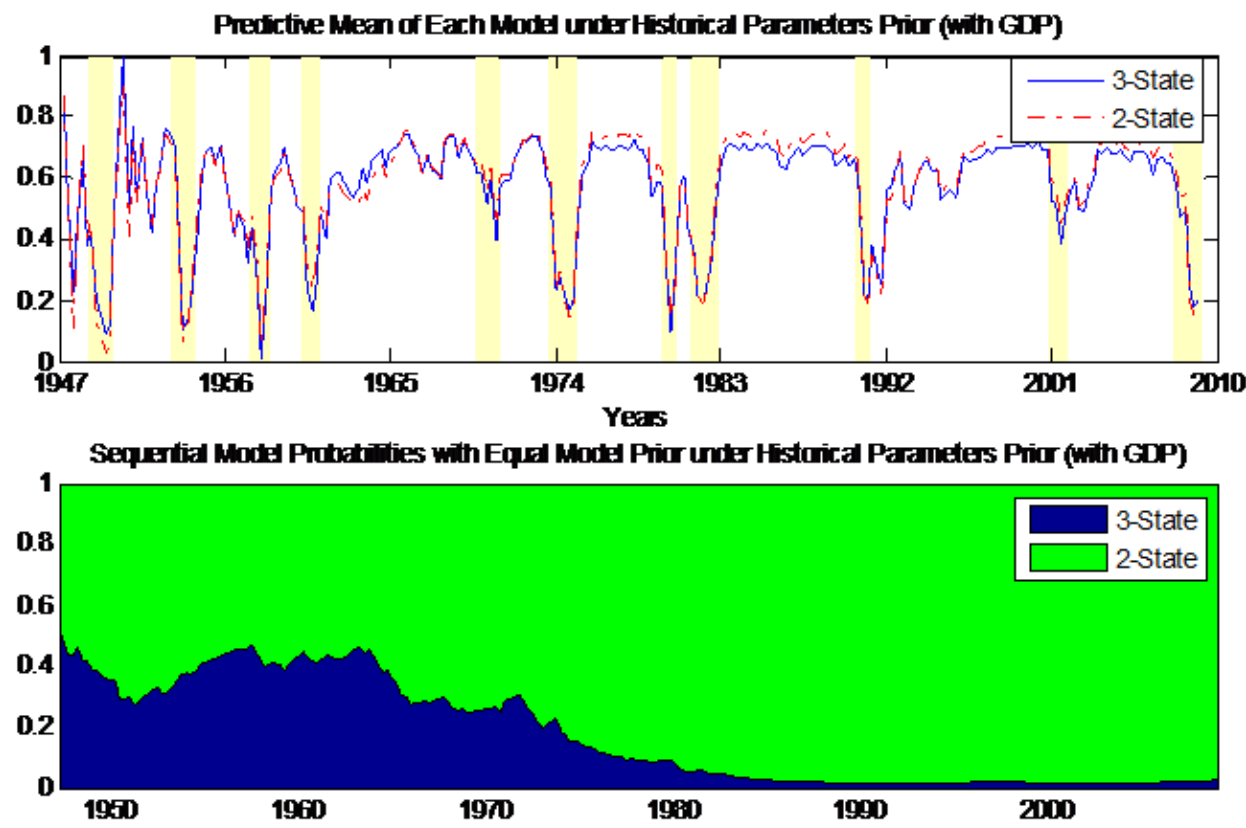

Predictine Mean unt: Fistorical Parancters Pria (with GDP)

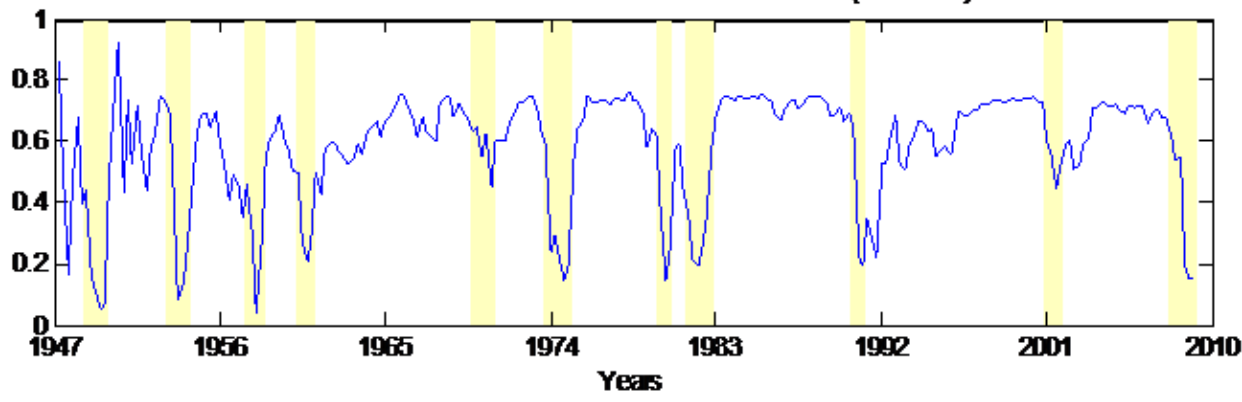

Figure 13: The top panel shows the quarterly conditional expected consumption growth, computed using the Historical Prior, from the three benchmark models: the "Merton model", and hidden 2and 3-state switching regime models. The "Merton model" is the 2-state i.i.d. model (a model with i.i.d. consumption growth but that allows for jumps). The middle plot shows the evolution of the probability of each model being the true model, where the prior at the beginning of the sample gives each model an equal probability. The lower plot shows the expected quarterly conditional consumption growth as perceived by an agent that also takes into account the model uncertainty (as given in the middle plot). The data in all cases go from 1947:Q2 - 2009:Q1. 


\section{Figure 14 - Conditional expected consumption growth (GDP)}
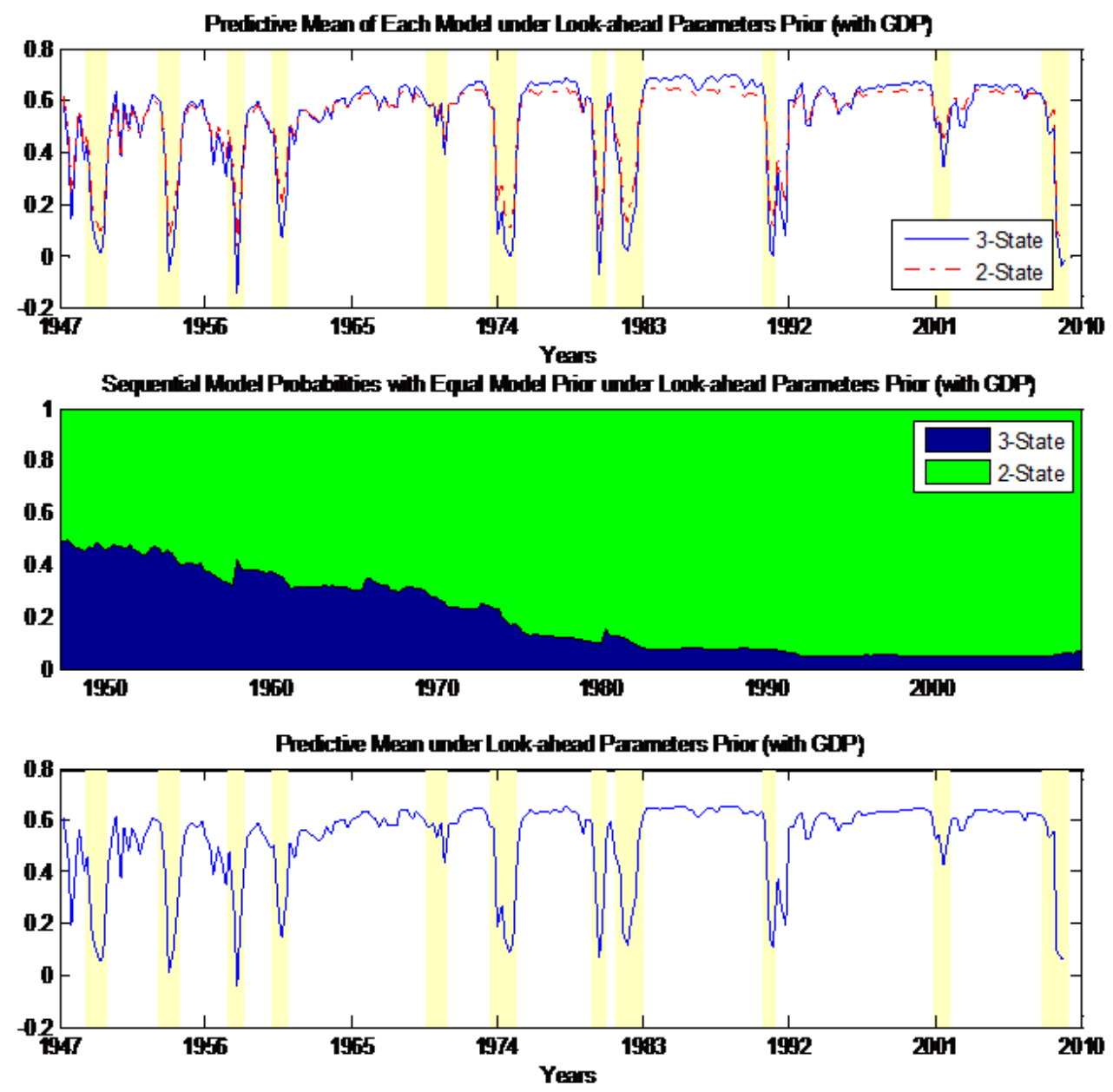

Figure 14: The top panel shows the quarterly conditional expected consumption growth, computed using the Rational Expectations Prior, from the three benchmark models: the Merton-model, and hidden 2- and 3-state switching regime models. The "Merton model" is the 2-state i.i.d. model (a model with i.i.d. consumption growth but that allows for jumps). The middle plot shows the evolution of the probability of each model being the true model, where the prior at the beginning of the sample gives each model an equal probability. The lower plot shows the expected quarterly conditional consumption growth as perceived by an agent that also takes into account the model uncertainty (as given in the middle plot). The data in all cases go from 1947:Q2 - 2009:Q1. 


\section{Table 2 - Updates in Beliefs versus Realized Stock Returns (GDP)}

Table 2: The table shows the results from regressions of innovations in agents' expectations of future consumption growth $\left(E_{t+1}\left[\Delta c_{t+2}\right]-E_{t}\left[\Delta c_{t+2}\right]\right)$ and conditional consumption growth variance $\left(\sigma_{t+1}^{2}\left[\Delta c_{t+2}\right]-\sigma_{t}^{2}\left[\Delta c_{t+2}\right]\right)$ versus contemporaneous excess stock market returns. In calculating the expectations, the parameter and model uncertainty is integrated out. The controls are lagged and contemporaneous realized log consumption growth, as well as the innovation in expected consumption growth derived from the 3-state model with fixed parameters (i.e., no model or parameter uncertainty). Both consumption and GDP data is used to estimate the models, as described in the main text. Panel A shows the results for the Historical priors, while Panel B shows the results for the Look-ahead priors. Heteroskedasticity and autocorrelation adjusted (Newey-West; 3 lags) standard errors are used. ${ }^{*}$ denotes significance at the $10 \%$ level, ${ }^{* *}$ denotes significance at the $5 \%$ level, and ${ }^{* * *}$ denotes significance at the 1\% level. The sample is from 1947:Q2 until 2009:Q1. In the below regressions, we have removed the first 40 observations (10 years), as a burn-in period to alleviate misspecification of the priors. However, similar results are obtained with no burn-in or with 80 quarter burn-in.

\begin{tabular}{|c|c|c|c|c|c|}
\hline \multicolumn{6}{|c|}{ Dependent variable: $r_{m, t+1}-r_{f, t+1}$ (excess market returns) } \\
\hline Panel A: Historical Prior & 1 & 2 & 3 & 4 & 5 \\
\hline$E_{t+1}\left[\Delta c_{t+2}\right]-E_{t}\left[\Delta c_{t+2}\right]$ & $\begin{array}{c}35.42^{* * *} \\
(5.74)\end{array}$ & $\begin{array}{c}37.25^{* * *} \\
(8.87)\end{array}$ & $\begin{array}{c}39.07^{* * *} \\
(8.16)\end{array}$ & & \\
\hline$\sigma_{t+1}^{2}\left[\Delta c_{t+2}\right]-\sigma_{t}^{2}\left[\Delta c_{t+2}\right]$ & & & & $\begin{array}{c}-65.80^{* * *} \\
(16.67)\end{array}$ & $\begin{array}{l}-28.99 \\
(22.37)\end{array}$ \\
\hline \multicolumn{6}{|l|}{ Controls: } \\
\hline$\Delta c_{t+1}$ & & $\begin{array}{l}-1.19 \\
(1.75)\end{array}$ & & & $\begin{array}{l}3.33^{*} \\
(1.81)\end{array}$ \\
\hline $\cos _{0}$ & & $\begin{array}{l}2.12 \\
(1.37)\end{array}$ & & & $\begin{array}{c}2.15 \\
(1.47)\end{array}$ \\
\hline$\left[E_{t+1}\left[\Delta c_{t+2}\right]-E_{t}\left[\Delta c_{t+2}\right]\right]$ Fixed parameters & & & $\begin{array}{l}-5.57 \\
(8.06)\end{array}$ & & \\
\hline$R_{a d j}^{2}$ & $14.7 \%$ & $15.1 \%$ & $14.5 \%$ & $6.1 \%$ & $9.9 \%$ \\
\hline Panel B: Look-ahead Prior & 1 & 2 & 3 & 4 & 5 \\
\hline$E_{t+1}\left[\Delta c_{t+2}\right]-E_{t}\left[\Delta c_{t+2}\right]$ & $\begin{array}{c}33.24^{* * *} \\
(5.46)\end{array}$ & $\begin{array}{c}30.11^{* * *} \\
(7.16)\end{array}$ & $\begin{array}{c}39.05^{* * *} \\
(7.74)\end{array}$ & & \\
\hline$\sigma_{t+1}^{2}\left[\Delta c_{t+2}\right]-\sigma_{t}^{2}\left[\Delta c_{t+2}\right]$ & & & & $\begin{array}{c}-69.71^{* * *} \\
(15.06)\end{array}$ & $\begin{array}{c}-45.47^{* *} \\
(21.08)\end{array}$ \\
\hline \multicolumn{6}{|l|}{ Controls: } \\
\hline$\Delta c_{t+1}$ & & $\begin{array}{c}0.15 \\
(1.51)\end{array}$ & & & $\begin{array}{c}2.33 \\
(1.84)\end{array}$ \\
\hline$\Delta c_{t}$ & & $\begin{array}{l}2.17^{*} \\
(1.35)\end{array}$ & & & $\begin{array}{l}2.50^{*} \\
(1.43)\end{array}$ \\
\hline$\left[E_{t+1}\left[\Delta c_{t+2}\right]-E_{t}\left[\Delta c_{t+2}\right]\right]$ Fixed parameters & & & $\begin{array}{l}-8.77 \\
(7.89)\end{array}$ & & \\
\hline$R_{a d j}^{2}$ & $14.2 \%$ & $14.8 \%$ & $14.1 \%$ & $7.9 \%$ & $11.1 \%$ \\
\hline
\end{tabular}


the agent has a preference for the timing of the resolution of uncertainty (i.e., if $\gamma \neq 1 / \psi$ ).

Our goal in this section is to, for reasonable preference parameters, understand how learning affects pricing relative to the benchmark case of fixed parameters. Given that the consumption dynamics are not ex post calibrated (in particular in the historical prior case) but estimated in real-time, we also do not calibrate preference parameters to match any particular moment(s). Instead, we simply use the preference parameters of Bansal and Yaron (2004). Thus, $\gamma=10, \psi=1.5$, and $\beta=0.998^{3}$.

Following both Bansal and Yaron (2004) and Lettau, Ludvigson, and Wachter (2008), we price a levered claim to the consumption stream with a leverage factor $\lambda$ of 4.5. The annual consumption volatility over the post-war sample is only $1.34 \%$, and so the systematic annual dividend volatility is therefore about 6\%. Quarterly log dividend growth is defined as:

$$
\Delta d_{t}=\lambda \Delta c_{t}+\varepsilon_{d, t}
$$

where $\varepsilon_{d, t} \stackrel{i . i . d .}{\sim} \mathcal{N}\left(-\frac{1}{2} \sigma_{d}^{2}, \sigma_{d}^{2}\right)$ is the idiosyncratic component of dividend growth. $\sigma_{d}$ is chosen to match the observed annual $11.5 \%$ volatility of dividend growth reported in Bansal and Yaron (2004). With these choices of $\lambda$ and $\sigma_{d}$ we also in fact closely match the sample correlation they report between annual consumption and dividend growth (0.55). ${ }^{23}$

With parameter and model uncertainty, the pricing problem becomes computationally infeasible due to the curse of dimensionality. As an example, the 3-state model has 12 parameters, which each have beliefs governed by two hype-parameters that will act as state variables. Therefore, we consider an anticipated utility approach proposed by Kreps (1997) as an approximation. The agent still treats the parameters and model probabilities as random variables when they update their beliefs, but as constants when they form expectations and make decisions. At each time $t$ the current posterior means are used as the values for the parameters and model probabilities when pricing assets. ${ }^{24}$ Thus, model and parameter

\footnotetext{
${ }^{23}$ The dividend dynamics imply that consumption and dividends are not cointegrated, which is a common assumptin (e.g., Campbell and Cochrane (1999), and Bansal and Yaron (2004)). One could impose cointegration between consumption and dividends, but at the cost of an additional state variable. Further, it is possible to also learn about $\lambda$ and $\sigma_{d}^{2}$. However, quarterly dividends are highly seasonal, which would severely complicate such an analysis. Further, data on stock repurchases is mainly annual. We leave a rigourous treatment of these issues to future research.

${ }^{24}$ The model is solved numerically through value function iteration at each time $t$ in the sample, conditional on the mean parameter beliefs at time $t$, which gives the time $t$ asset prices. The state variables when solving this model are the beliefs about the hidden states of the economy for each model under consideration. We consider only the model uncertainty between the 2- and 3-state models as the i.i.d. model is so strongly and quickly rejected in the data. For a detailed description of the model solution algorithm, please refer to the Appendix.
} 
uncertainty are not priced ex ante, although we do account for state uncertainty which also has been extensively analyzed in previous literature (see the Appendix for a brief review). This corresponds to a world where investors understand and account for business cycle fluctuations, but where they simply use their best guess for the parameters governing these dynamics. It would be computationally feasible to account for model uncertainty or to focus on parameter uncertainty over one (perhaps two) of the parameters, but we leave such considerations for future research.

\subsection{Unconditional Moments}

Table 3 reports realized asset pricing moments in the data, and also those generated by our agent who prices equity at each time period. The first 10 years are removed as a burn-in period to reduce concerns with regards to prior misspecification. We consider cases with and without parameter learning.

The models with parameter uncertainty match the observed equity premium reasonably well: $4.7 \%$ in the data versus $3.7 \%$ and $3.5 \%$ for the consumption only historical and lookahead priors, respectively. The models where GDP is used as an additional signal, which as reported earlier have a more severe recession state, have average sample excess equity returns of $4.4 \%$ and $4.2 \%$ for the historical and the look-ahead priors, respectively. This compares favorably to the benchmark fixed parameters two and three-state models which sample equity premiums are $1.5 \%$ and $1.8 \%$, respectively. Thus, allowing for parameter uncertainty more than doubles the sample risk premiums, despite the fact that parameter and model uncertainty are not priced risk factors in the anticipated utility pricing framework. The high sample equity premium arises because of the specific time path of beliefs, which we discuss next.

The table also reports the average ex ante equity risk premium $\left(E_{T}\left[E\left(R_{m, t+1}^{\text {excess }}\right) \mid I_{t}\right]\right.$, where $I_{t}$ denotes the information set (beliefs) of agents at time $t$ and $E_{T}[\cdot]$ denotes the sample average). The cases with parameter and model learning have about the same $e x$ ante risk premium. This implies that more than half of the excess returns achieved in these models occur due to ex post positive surprises in updates of beliefs. This is one of the primary implications of learning for this sample. Interestingly, after the burn-in period, this

Cogley and Sargent (2009) argue that anticipated utility approach is a close approximation to the true Bayesian approach, although their analysis is with respect to time-separable preferences. Piazzesi and Schneider (2010) is an example of a recent application of an anticipated utility pricing framework with Epstein-Zin preferences. 
effect is also strong in the look-ahead prior. With parameter and model uncertainty, agents beliefs quickly deviate from their full sample estimates, highlighting the difficulty of learning in real-time, similar to the problem faced by an econometrician.

The fact that the pricing results obtained from the look-ahead prior (after the burn-in period) are similar to the historical prior demonstrates that the sequence of shocks realized over the post-war sample generate a times series of beliefs that have a systematic time series pattern. In particular, the initial low mean and high volatility of consumption growth causes an upward revision in the mean growth rates and a negative revision in the volatility parameters, as described in Section 3. Fama and French (2002) reach a similar conclusion in terms of the ex post versus the ex ante risk premium when looking at the time-series of the aggregate price-earnings and price-dividend ratios. Sargent and Cogley (2008) assume negatively biased beliefs in their model to highlight the same mechanism. The results we present here are consistent with their conclusions, but our models are estimated from fundamentals alone.

The equity return volatility is, in all the cases permitting parameter and model uncertainty, very close to the $17.1 \%$ annual return volatility in the data (from $16.0 \%$ to $17.7 \%$ ). In contrast, the equity return volatility in the models with fixed parameters is about $12 \%$, which is almost all cash flow volatility as the annual dividend growth volatility is $11.5 \%$. Thus, the sample variation in discount and growth rates arising from updates in agents' beliefs cause excess return volatility (Shiller, 1980). This is reflected in the sample volatility of the log price-dividend ratio, which is 0.38 in the data. In the cases with parameter and model uncertainty the volatility of the log price-dividend ratio lies between 0.18 and $0.29 .{ }^{25}$ While this is only about $50 \%-75 \%$ of the volatility in the data, it is 3 to 5 times the volatility of the log price-dividend ratio in the benchmark fixed parameters models (here the volatility of the log price-dividend ratio is 0.06 for the two-state model and 0.07 for the three-state model).

The sample correlation between the log price-dividend ratios from the model versus the data, is 0.58 and 0.50 for the models using both GDP and consumption to estimate beliefs and 0.34 and 0.35 for the models using consumption only to estimate beliefs. The models with fixed parameters have lower correlations, 0.24 for the two-state model and 0.25 for the three-state model. As an alternative measure of the fit between the time-series of the sample

\footnotetext{
${ }^{25}$ The price-dividend ratio in each model is calculated as the corresponding in the data by summing the last four quarters of payouts to get annual payout. The price-dividend ratio from the data includes share repurchases in its definition of total dividends.
} 
price-level in the data versus those in the models considered here, the highest covariance between the price-dividend ratio in the data and the models with parameter and model uncertainty is 0.0551 , whereas the highest covariance between the price-dividend ratio in the data and the models with fixed parameters is 0.0067 - a difference close to an order of magnitude. Thus, with parameter and model learning the model tracks the aggregate stock market price level (normalized by dividends) much more closely than either of the models we consider with fixed parameters. The price-level, a first order moment, is arguably even more important than matching the second order moments that usually are the focus in asset pricing.

\section{Table 3 - Asset Price Moments}

Table 3: The table reports the asset pricing implications of the models with an anticipated utility version of the Epstein-Zin preferences under different priors, as well as the fixed parameters cases. For all the models, $\gamma=10, \beta=0.994, \psi=1.5, \lambda=4.5$. The volatility of the idiosyncratic component of dividend growth $\left(\epsilon_{d, t}\right)$ is calibrated to match the historical standard deviation of dividend growth, as reported in Bansal and Yaron (2004). The statistics are annualized. The expectation operator with a $T$ subscript, $E_{T}$, denotes the sample average, while the volatility operator, $\sigma_{T}$ denotes the sample standard deviation. 'Cons. only' denotes the model results in the case where only consumption growth is used to update beliefs, while 'Cons. + GDP' denotes the model results in the case where both consumption and GDP growth are used to update beliefs. The full sample period is from 1947:Q2 until 2009:Q1. However, we have removed the first 40 observations (10 years), as a burn-in period to alleviate misspecification of the priors. Similar results are obtained with no burn-in or with 80 quarter burn-in.

\begin{tabular}{|c|c|c|c|c|c|c|c|}
\hline \multirow[b]{2}{*}{ Moments } & \multirow{2}{*}{$\begin{array}{c}\text { Data } \\
\text { 1957:Q2- } \\
\text { 2009:Q1 }\end{array}$} & \multicolumn{2}{|c|}{ Historical prior } & \multicolumn{2}{|c|}{ Look-ahead prior } & \multicolumn{2}{|c|}{ Fixed parameters } \\
\hline & & $\begin{array}{l}\text { Cons. } \\
\text { only }\end{array}$ & $\begin{array}{l}\text { Cons. }+ \\
\text { GDP }\end{array}$ & $\begin{array}{l}\text { Cons. } \\
\text { only }\end{array}$ & $\begin{array}{l}\text { Cons. }+ \\
\text { GDP }\end{array}$ & $\begin{array}{l}2 \text {-state } \\
\text { model }\end{array}$ & $\begin{array}{l}3 \text {-state } \\
\text { model }\end{array}$ \\
\hline \multicolumn{8}{|l|}{ The real risk-free rate: } \\
\hline$E_{T}\left(r_{t}^{f}\right)$ & $1.6 \%$ & $3.7 \%$ & $3.7 \%$ & $3.7 \%$ & $3.6 \%$ & $3.7 \%$ & $3.7 \%$ \\
\hline$\sigma_{T}\left(r_{t}^{f}\right)$ & $1.6 \%$ & $1.0 \%$ & $1.2 \%$ & $0.8 \%$ & $1.0 \%$ & $0.7 \%$ & $0.8 \%$ \\
\hline \multicolumn{8}{|c|}{ The dividend claim: $d_{t}=\lambda c_{t}+\varepsilon_{d, t}$} \\
\hline ex post: & & & & & & & \\
\hline$E_{T}\left(r_{t}-r_{t}^{f}\right)$ & $4.7 \%$ & $3.8 \%$ & $4.4 \%$ & $3.5 \%$ & $4.2 \%$ & $1.5 \%$ & $1.8 \%$ \\
\hline$\sigma_{T}\left(r_{t}-r_{t}^{f}\right)$ & $17.1 \%$ & $17.9 \%$ & $17.0 \%$ & $16.3 \%$ & $16.1 \%$ & $12.2 \%$ & $12.4 \%$ \\
\hline Sharpe ratio & 0.27 & 0.21 & 0.26 & 0.21 & 0.26 & 0.12 & 0.14 \\
\hline$\sigma_{T}\left(p d_{t}\right)$ & 0.38 & 0.19 & 0.23 & 0.21 & 0.29 & 0.06 & 0.07 \\
\hline $\operatorname{Corr}_{T}\left(p d_{t}^{\text {Model }}, p d_{t}^{\text {Data }}\right)$ & $n / a$ & 0.34 & 0.58 & 0.35 & 0.50 & 0.24 & 0.25 \\
\hline $\begin{array}{l}\text { ex ante: } \\
E_{T}\left[E_{t}\left(r_{t+1}-r_{t+1}^{f}\right)\right]\end{array}$ & $n / a$ & $1.9 \%$ & $2.5 \%$ & $1.5 \%$ & $1.8 \%$ & $1.5 \%$ & $1.8 \%$ \\
\hline
\end{tabular}




\section{Figure 15 - Price-Dividend Ratios}

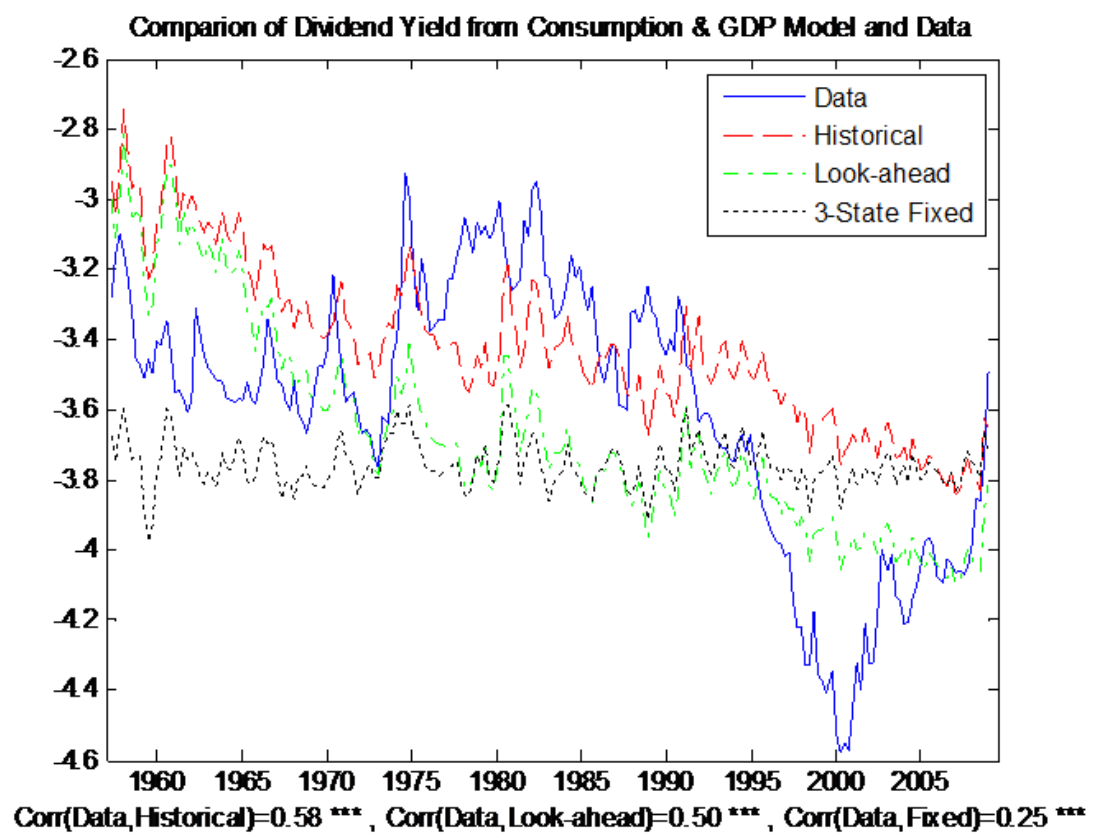

Figure 15: The figures shows the time series of the annualized log dividend-price ratios of the equity claim obtained from the anticipated utility Epstein-Zin models where beliefs are based on observing consumption and GDP growth. As benchmarks, the log dividend-price ratio from the 3-state fixed parameters model and the data are also displayed.

Figure 15 shows the dividend yields implied by the models as well as that in the data for the cases where beliefs are updated using both consumption and GDP growth. As can be seen from the graph, the dividend yield from the fixed parameter benchmark cases barely displays any time-variation, while the models with parameter uncertainty do a lot better. Also, note that only when we account for parameter and model uncertainty is there a downward trend over the sample in the log dividend-price ratios similar to that we observe in the data. Recessions are, as in the data, associated with dips in the price-dividend ratios in all the models, although these are barely perceptible in the benchmark, fixed parameters model. Notice also the sharp increase in the log dividend-price ratio of the model using the historical prior at the onset of the financial crisis in 2008 - a consequence of the disaster state in this model which is closest to the experience of the Great Depression. 


\subsection{Return Predictability}

Lastly, we consider excess market return forecasting regression using the dividend yield as the predictive variable. These regressions have a long history in asset pricing and remain a feature of the data that asset pricing models typically aim to explain (e.g., Campbell and Cochrane (1999), Bansal and Yaron (2004)). However, the strength of the empirical evidence is under debate (see, e.g., Stambaugh (1999), Ang and Bekaert (2007), Boudoukh, Richardson and Whitelaw (2008), and Goyal and Welch (2008) for critical analyses). Here we run standard forecasting regressions overlapping at the quarterly frequency using the sample of market returns and dividend yields as implied by each of the models. Note that, as before, we are not looking at population moments or average small-sample moments, but the single sample generated by feeding the models the actual sample of realized consumption growth.

Table 4 shows the forecasting regressions over different return forecasting horizons from the data. We use both the market dividend yield and the approximation to the consumptionwealth ratio, cay, of Lettau and Ludvigson (2001) to show the amount of predictability implied by these regressions in the data. We then run the same regressions using model implied returns and dividend yields. The benchmark models with fixed parameters (bottom right in the table) show no evidence of return predictability at the $5 \%$ significance level and the $R^{2}$ 's are very small. These models do, in fact, feature time-variation in the equity risk premium, but the standard deviation of the risk premiums are only about $0.5 \%$ per year and so the signal-to-noise ratio in these regressions is too small to result in significant predictability in a sample of the length we consider here. The models with parameter uncertainty, however, display significant in-sample return predictability and the regression coefficients and the $R^{2}$ 's are large and increasing in the forecasting horizon similar to those in the data. The ex ante predictability in these models is in fact similar to that in the fixed parameters cases, but since the parameters are updated at each point in time, there is significant ex post predictability. For instance, an increase in the mean parameters of consumption growth leads to high returns and lower dividend yield. Thus, a high dividend yield in sample forecasts high excess returns in sample. This is the same effect of learning as that pointed out in Timmermann (1993) and Lewellen and Shanken (2002). The models here show that the significant regression coefficients in the classical forecasting regressions show up in the sample only in the model where there is parameter learning which generates

a significant difference between ex ante expected returns and ex post realizations. Thus, the 
model predicts that the amount of predictability is much smaller out-of-sample, consistent with the empirical evidence in Goyal and Welch (2008) and Ang and Bekaert (2007).

\section{Conclusion}

Typical implementations of rational expectations, consumption-based exchange economy models assume the agents know - either by endowment or via learning - the data generating process for aggregate consumption growth. This paper studies the statistical problem and asset pricing implications of learning about parameters, state variables, and models in a standard model of consumption growth. Our approach focuses not on the general implications (e.g., population properties) of learning, but on the specific implications generated by learning about U.S. consumption dynamics using the post World War II period.

We show that the agents' beliefs about the consumption growth dynamics are strongly time-varying, nonstationary, and different from the benchmark models where parameters are fixed and known. This generates a number of important implications. First, via learning, our agent is able to detect non-i.i.d. or persistent components of consumption dynamics in real time. This has important asset pricing implications with standard preferences such as Epstein-Zin. Second, the agent's beliefs are highly non-stationary, as beliefs about parameters or moments can trend up or down during the sample. This leads to, for example, a difference between ex post and ex ante risk premiums, return volatility, and return predictability. Third, revisions in beliefs about the conditional mean of consumption growth are positively and significantly related to realized stock returns, as one would expect if agents in reality learn about these aspects of the economy. Updates in the objective expectations (obtained using full sample parameter estimates and treating these as known to the agent), on the other hand, are not significantly related to stock returns when used as a control in these regressions. We view this as strong evidence that model and parameter learning are important for understanding the joint dynamics of aggregate consumption and asset returns.

In terms of asset pricing implications, we find that up to half of the equity premium in the post-WW2 sample is a consequence of positive revisions in agents' beliefs about the aggregate consumption dynamics. Further, most of the in sample significant evidence of excess market return predictability is a result of ex post overfitting. This is not to say that the ex ante price of risk and risk-premium are not time-varying - we estimate that they are also in our setting - the conditional Sharpe ratio and risk premium of aggregate equity 


\section{Table 4 - Return Forecasting Regressions}

Table 4: This table presents quarterly excess market return forecasting regressions over various forecasting horizons ( $q$ quarters; 1 to 16 ). The top right shows the results when using market data and a measure of the log aggregate dividend yield; the cay-variable of Lettau and Ludvigsson (2001) and the CRSP aggregate log dividend yield $\left(\ln \frac{D_{t}}{P_{t}}\right.$ where dividends are measured as the sum of the last four quarters' dividends. The rest of the table shows the results using the returns and dividend yield generated within the models based on the Historical priors, the Look-ahead priors, and the fixed parameter case. "Cons. only" denotes the model results in the case where only consumption growth is used to update beliefs, while "Cons. and GDP" denotes the model results in the case where both consumption and GDP growth are used to update beliefs. NeweyWest autocorrelation and heteroskedasticity adjusted standard errors are given in parentheses (the number of lags is equal to the number of overlapping observations). ${ }^{*}$ denotes significance at the $10 \%$ level, ${ }^{* *}$ denotes significance at the $5 \%$ level, and ${ }^{* * *}$ denotes significance at the $1 \%$ level. The full sample period is from 1947:Q2 until 2009:Q1. However, we have removed the first 40 observations (10 years), as a burn-in period to alleviate misspecification of the priors. Similar results are obtained with no burn-in or with 80 quarter burn-in.

\begin{tabular}{|c|c|c|c|c|c|c|c|c|}
\hline \multirow[b]{3}{*}{$q$} & \multicolumn{8}{|c|}{$r_{t, t+q}-r_{f, t, t+q}=\alpha_{q}+\beta_{q, d p} \ln \left(D_{t} / P_{t}\right)+\varepsilon_{t, t+q}$} \\
\hline & \multicolumn{2}{|c|}{$\ln \left(D_{t} / P_{t}\right):=\operatorname{cay}_{t}$} & \multicolumn{2}{|c|}{$\ln \left(D_{t} / P_{t}\right):=\ln \frac{\Sigma_{j=0}^{3} D_{t-j}^{M k t .}}{P^{M k t .}}$} & \multicolumn{2}{|c|}{ Cons. only } & \multicolumn{2}{|c|}{ Cons. and GDP } \\
\hline & $\beta_{d p}($ s.e. $)$ & $R_{a d j}^{2}$ & $\beta_{d p}($ s.e. $)$ & $R_{a d j}^{2^{t}}$ & $\beta_{d p}($ s.e. $)$ & $R_{a d j}^{2}$ & $\beta_{d p}($ s.e. $)$ & $R_{a d j}^{2}$ \\
\hline 1 & $\begin{array}{c}1.19^{* * *} \\
(0.31)\end{array}$ & $4.67 \%$ & $\begin{array}{l}0.03^{*} \\
(0.02)\end{array}$ & $1.6 \%$ & $\begin{array}{c}0.05 \\
(0.03)\end{array}$ & $1.2 \%$ & $\begin{array}{c}0.03 \\
(0.02)\end{array}$ & $1.3 \%$ \\
\hline 4 & $\begin{array}{c}4.29^{* * *} \\
(1.18)\end{array}$ & $15.65 \%$ & $\begin{array}{l}0.11^{* *} \\
(0.05)\end{array}$ & $6.6 \%$ & $\begin{array}{c}0.23^{* * *} \\
(0.08)\end{array}$ & $7.1 \%$ & $\begin{array}{c}0.15^{* *} \\
(0.055)\end{array}$ & $6.7 \%$ \\
\hline 8 & $\begin{array}{c}7.60^{* * *} \\
(1.72)\end{array}$ & $28.1 \%$ & $\begin{array}{l}0.17^{*} \\
(0.10)\end{array}$ & $8.5 \%$ & $\begin{array}{c}0.47^{* * *} \\
(0.13)\end{array}$ & $16.6 \%$ & $\begin{array}{l}0.28^{* * *} \\
(0.078)\end{array}$ & $13.2 \%$ \\
\hline \multirow[t]{3}{*}{16} & $\begin{array}{c}12.31^{* * *} \\
(1.82)\end{array}$ & $41.6 \%$ & $\begin{array}{l}0.22^{* *} \\
(0.11)\end{array}$ & $9.5 \%$ & $\begin{array}{c}0.71^{* * *} \\
(0.18)\end{array}$ & $25.8 \%$ & $\begin{array}{c}0.42^{* * *} \\
(0.13)\end{array}$ & $16.9 \%$ \\
\hline & \multicolumn{4}{|c|}{ Look-ahead prior } & \multicolumn{4}{|c|}{ Fixed parameters } \\
\hline & $\begin{array}{r}\text { Cons. } \\
\beta_{d p} \text { (s.e.) }\end{array}$ & $\begin{array}{l}\text { only } \\
R_{a d j}^{2}\end{array}$ & $\begin{array}{l}\text { Cons. } \\
\beta_{d p} \text { (s.e.) }\end{array}$ & $\begin{array}{l}\text { GDP } \\
R_{a d j}^{2}\end{array}$ & $\begin{array}{l}\text { 2-state 1 } \\
\beta_{d p} \text { (s.e.) }\end{array}$ & $\begin{array}{l}\text { odel } \\
R_{a d j}^{2}\end{array}$ & $\begin{array}{r}\text { 3-state } \\
\beta_{d p} \text { (s.e.) }\end{array}$ & $\begin{array}{l}\text { odel } \\
R_{a d j}^{2}\end{array}$ \\
\hline 1 & $\begin{array}{c}0.038 \\
(0.030)\end{array}$ & $1.1 \%$ & $\begin{array}{c}0.03 \\
(0.03)\end{array}$ & $0.7 \%$ & $\begin{array}{l}-0.01 \\
(0.062)\end{array}$ & $0.0 \%$ & $\begin{array}{c}0.004 \\
(0.062)\end{array}$ & $0.0 \%$ \\
\hline 4 & $\begin{array}{l}0.20^{* *} \\
(0.07)\end{array}$ & $7.1 \%$ & $\begin{array}{l}0.13^{* *} \\
(0.07)\end{array}$ & $5.4 \%$ & $\begin{array}{c}0.19 \\
(0.17)\end{array}$ & $1.0 \%$ & $\begin{array}{c}0.20 \\
(0.16)\end{array}$ & $1.2 \%$ \\
\hline 8 & $\begin{array}{c}0.44^{* * *} \\
(0.12)\end{array}$ & $16.0 \%$ & $\begin{array}{l}0.26^{* *} \\
(0.09)\end{array}$ & $10.5 \%$ & $\begin{array}{l}0.37^{*} \\
(0.24)\end{array}$ & $2.3 \%$ & $\begin{array}{l}0.41^{*} \\
(0.23)\end{array}$ & $2.7 \%$ \\
\hline 16 & $\begin{array}{c}0.65^{* * *} \\
(0.17)\end{array}$ & $21.8 \%$ & $\begin{array}{l}0.39^{* *} \\
(0.17)\end{array}$ & $14.3 \%$ & $\begin{array}{c}0.26 \\
(0.31)\end{array}$ & $0.7 \%$ & $\begin{array}{c}0.28 \\
(0.30)\end{array}$ & $0.8 \%$ \\
\hline
\end{tabular}


returns are counter-cyclical in all the models. For the models with fixed parameters, however, the variation in the conditional risk premium is too small relative to the volatility of realized equity returns to result in significant return predictability in the sample at hand. Finally, the covariance between the price-dividend ratios in the models that feature parameter and model learning and the market price-dividend ratio in the data are an order of magnitude higher than the covariance between the market price-dividend ratio and the price-dividend ratios obtained from the benchmark models with fixed parameters. The improvement in the fit of the time series of the price-dividend ratio is due to both higher volatility of the price dividend ratio in the models with parameter learning and higher correlation with the price-dividend ratio in the data. In sum, the evidence presented in this paper indicates that parameter and model learning help us understand many of the quantitative puzzles posed by the joint dynamics of aggregate consumption growth and asset prices and returns observed in the U.S. post-WW2 sample.

\section{References}

[1] Ai, H. (2010), "Information about Long-Run Risk: Asset Pricing Implications," forthcoming, Journal of Finance.

[2] Ang, A. and G. Bekaert (2007), "Return predictability: Is it there?", Review of Financial Studies, 20, 3, 651-707.

[3] Backus, D., M. Chernov and I. Martin (2009), "Disasters Implied by Equity Index Options", NYU Working Paper

[4] Bakshi, G. and G. Skoulakis (2010), "Do Subjective Expectations Explain Asset Pricing Puzzles?", Journal of Financial Economics, December 2010, 117 - 140.

[5] Bansal, R. and A. Yaron (2004), "Risks for the Long-Run: A Potential Resolution of Asset Pricing Puzzles", Journal of Finance 59(4), 1481 - 1509

[6] Bansal, R. and I. Shaliestovich (2010), "Confidence Risk and Asset Prices," American Economic Review PEP, 100, 537 - 541.

[7] Barberis, N. (2000), "Investing for the Long Run When Returns Are Predictable", Journal of Finance 55(1), 225 - 264 
[8] Barillas, F., Hansen, L. and T. Sargent, "Doubts or Variability?", Journal of Economic Theory, forthcoming.

[9] Barro, R. (2006), "Rare Disasters and Asset Markets in the Twentieth Century", Quarterly Journal of Economics 121, 823 - 866

[10] Barro, R., Nakamura, E., Steinsson, J., and J. Ursua (2009), "Crises and Recoveries in an Empirical Model of Consumption Disasters," Columbia Business School working paper

[11] Barro, R. and J. Ursua (2008), "Consumption Disasters since 1870", Brookings Papers on Economic Activity

[12] Bhamra, H., L. Kuehn and I. Strebulaev (2009), "The Levered Equity Risk Premium and Credit Spreads: A Unified Framework", Review of Financial Studies (forthcoming)

[13] Boguth, O. and L. Kuehn (2009), "Consumption Volatility Risk," Working Paper Carnegie Mellon University

[14] Boudoukh, J., M. Richardson, and R. Whitelaw (2008), "The Myth of Long Horizon Predictability," Review of Financial Studies 21, 1577 - 1605.

[15] Brandt, M. , Q. Zeng, and L. Zhang (2004), "Equilibrium stock return dynamics under alternative rules of learning about hidden states," Journal of Economic Dynamics and Control, 28, 1925 - 1954.

[16] Bray, M. M. and N. E. Savin (1986), "Rational Expectations Equilibria, Learning, and Model Specification," Econometrica 54, 1129 - 1160.

[17] Cagetti, M., L. Hansen, T. Sargent and N. Williams (2002), "Robustness and Pricing with Uncertain Growth", Review of Financial Studies 15, 363 - 404

[18] Carvalho, Johannes, Lopes, and Polson (2010a), "Particle Learning and Smoothing," Statistical Science, 25, 88-106.

[19] Carvalho, Johannes, Lopes, and Polson (2010b - forthcoming), "Particle learning: Simulation-based Bayesian inference," Bayesian Statistics 9.

[20] Cecchetti, S., P. Lam and N. Mark (1990), "Mean Reversion in Equilibrium Asset Prices", American Economic Review 80, 398 - 418 
[21] Cecchetti, S., P. Lam and N. Mark (1993), "The Equity Premium and the Risk Free Rate: Matching the Moments", Journal of Monetary Economics 31, 21 - 46

[22] Chen, H. (2009), "Macroeconomic Conditions and the Puzzles of Credit Spreads and Capital Structure", Journal of Finance (forthcoming)

[23] Chen, H., S. Joslin, and N. Tran (2010), "Rare Disasters and Risk Sharing with Heterogeneous Beliefs," MIT Working paper

[24] Cogley, T. and T. Sargent (2008), "The Market Price of Risk and the Equity Premium: A Legacy of the Great Depression?", Journal of Monetary Economics 55, 454 - 476

[25] Cogley, T. and T. Sargent (2009), "Anticipated Utility and Rational Expectations as Approximations of Bayesian Decision Making", International Economic Review 49, 185 $-221$

[26] David, A. and P. Veronesi (2009), "What ties return volatilities to price valuations and fundamentals?" University of Calgary Working Paper.

[27] Detemple, J. (1986), "Asset pricing in a production economy with incomplete information." Journal of Finance, 41, 383-390.

[28] Dothan, M. U. and D. Feldman (1986), "Equilibrium interest rates and multiperiod bonds in a partially observable economy." Journal of Finance, 41, 369-382.

[29] Drechsler, I. and A. Yaron (2008), "What's Vol Got to Do with It?", Working Paper, Wharton School of Business, University of Pennsylvania

[30] Epstein, L. and S. Zin (1989), "Substitution, Risk Aversion, and the Temporal Behavior of Consumption and Asset Returns: A Theoretical Framework", Econometrica 57, 937 - 969

[31] Fama, E. and K. French (1988), "Dividend yields and expected stock returns," Journal of Financial Economics 22, 3 - 25.

[32] Froot, K. and S. Posner (2002), "The Pricing of Event Risks with Parameter Uncertainty", GENEVA Papers on Risk and Insurance - Theory 27, 153 - 165 
[33] Hall, R. (1978), "Stochastic Implications of the Life Cycle-Permanent Income Hypothesis: Theory and Evidence," Journal of Political Economy, December 1978, 86(6), pp. 971-987.

[34] Gabaix, X. (2009), "Variable Rare Disasters: An Exactly Solved Framework for Ten Puzzles in Macro-Finance", $N Y U$ Working Paper

[35] Gennotte, G. (1986), "Optimal Portfolio Choice under Incomplete Information," Journal of Finance, 61, 733-749.

[36] Geweke, J. (2001), "A note on some limitations of CRRA utility," Economics Letters $71,341-345$.

[37] Goyal A. and I. Welch, "A Comprehensive Look at the Empirical Performance of Equity Premium Prediction," July 2008, Review of Financial Studies 21(4) 1455-1508.

[38] Hansen, L. (2007), "Beliefs, Doubts and Learning: Valuing Macroeconomic Risk," Richard T. Ely Lecture, The American Economic Review 97, No. 2., 1 - 30

[39] Hansen, L. and T. Sargent (2009), "Robustness, Estimation and Detection," Working paper.

[40] Hansen, L. and T. Sargent (2010), "Fragile Beliefs and the Price of Uncertainty," Quantitative Economics, Vol. 1, Issue 1, pp. 129-162

[41] Johannes, M. and N. Polson (2006), "Particle Filtering," Springer Verlag Handbook of Financial Time Series, edited by Torben G. Andersen Richard A. Davis, Jens-Peter Kreiss, and Thomas Mikosch, September 2006.

[42] Kandel, S. and R. Stambaugh (1990), "Expectations and Volatility of Consumption and Asset Returns", Review of Financial Studies 2, 207 - 232

[43] Kandel, S. and R. Stambaugh (1996), "On the Predictability of Stock Returns: An Asset-Allocation Perspective", Journal of Finance 51, 66 - 74

[44] Kreps, D. (1998), "Anticipated Utility and Dynamic Choice", Frontiers of Research in Economic Theory, (Cambridge: Cambridge University Press) 242 - 274

[45] Lettau, M. and S. Ludvigson (2001), "Consumption, Aggregate Wealth and Stock Returns," Journal of Finance 56, 815 - 849 
[46] Lettau, M., S. Ludvigson and J. Wachter (2008), "The Declining Equity Premium: What Role Does Macroeconomic Risk Play?", Review of Financial Studies 21(4), 1653 $-1687$

[47] Lewellen, J. and J. Shanken (2002), "Learning, Asset-Pricing Tests, and Market Efficiency," Journal of Finance (57(3), 1113 - 1145.

[48] Lubos, P. (2000), "Portfolio Selection and Asset Pricing Models," Journal of Finance $55,179-223$

[49] Lucas, R. (1978), "Asset Prices in an Exchange Economy," Econometrica 46, 1429-1446

[50] Lucas, R. and T. Sargent (1979), "After Keynesian Macroeconomics," The Federal Reserve Bank of Minneapolis, Quarterly Review 321.

[51] Mehra, R. and E. Prescott (1985), "The Equity Premium: A Puzzle", Journal of Monetary Economics 15, 145 - 161

[52] Moore, B. and H. Schaller (1996), "Learning, Regime Switches, and Equilibrium Asset Pricing Dynamics", Journal of Economic Dynamics and Control 20, 979 - 1006

[53] Pastor, L. and P. Veronesi (2003), "Stock valuation and learning about profitability," Journal of Finance, 58, 1479 - 1789.

[54] Pastor, L. and P. Veronesi (2006), "Was there a NASDAQ bubble in the late 1990s?," Journal of Financial Economics, 81, 61 - 100.

[55] Pastor, L. and P. Veronesi (2009), "Learning in Financial Markets," Annual Review of Financial Economics.

[56] Piazzesi, M. and M. Schneider (2010), "Trend and Cycle in Bond Risk Premia," Working Paper Stanford University

[57] Rietz, T. (1988), "The equity risk premium: A solution?", Journal of Monetary Economics, Volume 22, Issue 1, July 1988, Pages 117-131

[58] Romer, C. (1989), "The Prewar Business Cycle Reconsidered: New Estimates of Gross National Product, 1869-1908", Journal of Political Economy 97, 1 - 37 
[59] Shaliastovich, I. (2008), "Learning, Confidence and Option Prices," Working Paper, Duke University

[60] Stambaugh, R. (1999), "Predictive Regressions." Journal of Financial Economics, 1999, 54, pp. 375-421.

[61] Timmermann, A. (1993), "How Learning in Financial Markets Generates Excess Volatility and Predictability in Stock Prices." Quarterly Journal of Economics, 1993, 108, 1135-1145.

[62] Veronesi, P. (1999), "Stock Market Overreaction to Bad News in Good Times: A Rational Expectations Equilibrium Model," Review of Financial Studies, 12, 5, Winter 1999

[63] Veronesi, P. (2000), "How does information quality affect stock returns?" Journal of Finance, 55, .

[64] Weitzman, M. (2007), "Subjective Expectations and Asset-Return Puzzles," American Economic Review, 97(4), 1102-1130.

[65] Whitelaw, R. (2000), "Stock Market Risk and Return: An Equilibrium Approach", Review of Financial Studies 13, 521 - 547.

[66] Working, H. (1960), "Note on the Correlation of First Differences of Averages in a Random Chain," Econometrica 28(4), 916 - 918.

[67] Xia, Y. (2001), "Learning about Predictability: The Effects of Parameter Uncertainty on Dynamic Asset Allocation", Journal of Finance 56(1), 205 - 246

\section{Appendix}

\subsection{Existing literature and alternative approaches for parameter, state, and model uncertainty.}

Our paper is related to a large literature studying the asset pricing implications of parameter or state learning. Most of this literature focuses on learning about a single unknown parameter or state variable (assuming the other parameters and/or states are known) that determines dividend dynamics and power utility. For example, Timmerman (1993) considers 
the effect of uncertainty on the average level of dividend growth, assuming other parameters are known, and shows in simple discounted cash-flow setting that parameter learning generates excess volatility and patterns consistent with the predictability evidence (see also Timmerman 1996). Lewellen and Shanken (2002) study the impact of learning about mean cash-flow parameters with exponential utility with a particular focus on return predictability.

Veronesi (2000) considers the case of learning about mean-dividend growth rates in a model with underlying dividend dynamics with power utility and focuses on the role of signal precision or information quality. Pastor and Veronesi (2003, 2006) study uncertainty about a fixed dividend-growth rate or profitability levels with an exogenously specified pricing kernel, in part motivated in order to derive cross-sectional implications. Weitzman (2007) and Bakshi and Skoulakis (2009) consider uncertainty over volatility.

Cogley and Sargent (2008) consider a two-state Markov-switching model, parameter uncertainty over one of the transition probabilities, tilt beliefs to generate robustness via pessimistic beliefs, and use power utility. After calibrating the priors to the 1930s experience, they simulate data from a true model calibrated to the post War experience to show how priced parameter uncertainty and concerns for robustness impact asset prices, in terms of the finite sample distribution over various moments.

A number of papers consider state uncertainty, where the state evolves discretely via a Markov switching model or smoothing via a Gaussian process. Moore and Shaller (1996) consider consumption/dividend based Markov switching models with state learning and power utility. Brennen and Xia (2001) consider the problem of learning about dividend growth which is not a fixed parameter but a mean-reverting stochastic process, with power utility. Veronesi (2004) studies the implications of learning about a peso state in a Markov switching model with power utility. David and Veronesi (2010) consider a Markov switching model with learning about states.

In the case of Epstein-Zin utility, Brandt, Zeng, and Zhang (2004) consider alternative rules for learning about an unknown Markov state, assuming all parameters and the model is known. Lettau, Ludvigson, and Wachter (2008) consider information structures where the economic agents observe the parameters but learn about states in Markov switching consumption based asset pricing model. Chen and Pakos (2008) consider learning about the mean of consumption growth which is a Markov switching process. Ai (2010) studies learning in a production-based long-run risks model with Kalman learning about a persistent latent state variable. Bansal and Shaliastovich (2008) and Shaliastovich (2010) consider learning about the persistent component in a Bansal and Yaron (2004) style model with sub-optimal 
Kalman learning.

Additionally, some papers consider combinations of parameter or model uncertainty and robustness, see, e.g., Hansen and Sargent $(2000,2009)$ and Hansen (2008).

\subsection{Econometrics}

This section briefly reviews the mechanics of sequential Bayesian learning and introduces the econometric methods needed to solve the high-dimensional learning problem. For ease of exposition, we abstract here from the problem of model uncertainty and drop the dependence on the model specification. Model uncertainty can be dealt with easily in a fashion analogous to the problem considered here.

The agent begins with initial beliefs over the parameters and states, $p\left(\theta, s_{t}\right)=p\left(s_{t} \mid \theta\right) p(\theta)$, and then updates via Bayes' rule. If at time $t$ the agent holds beliefs $p\left(\theta, s_{t} \mid y^{t}\right)$, then updating occurs in a two step process by first computing the predictive distribution, $p\left(\theta, s_{t+1} \mid y^{t}\right)$, and then updating via the likelihood function, $p\left(y_{t+1} \mid s_{t+1}, \theta\right)$ :

$$
p\left(\theta, s_{t+1} \mid y^{t+1}\right) \propto p\left(y_{t+1} \mid \theta, s_{t+1}\right) p\left(\theta, s_{t+1} \mid y^{t}\right) .
$$

The predictive distribution is

$$
p\left(\theta, s_{t+1} \mid y^{t}\right)=\int p\left(s_{t+1} \mid s_{t}, \theta\right) p\left(\theta, s_{t} \mid y^{t}\right) d s_{t},
$$

which shows the recursive nature of Bayesian updating, as $p\left(\theta, s_{t+1} \mid y^{t+1}\right)$ is functionally dependent on $p\left(\theta, s_{t} \mid y^{t}\right)$.

The main difficulty is characterizing $p\left(\theta, s_{t} \mid y^{t}\right)$ for each $t$, which is needed for sequential learning. Unfortunately, even though $s_{t}$ is discretely valued, there is no analytical form for $p\left(\theta, s_{t} \mid y^{t}\right)$, as it is high-dimensional and the dependence on the data is complicated and nonlinear. We use Monte Carlo methods called particle filters to generate approximate samples from $p\left(\theta, s_{t} \mid y^{t}\right)$. Johannes and Polson (2008) developed the general approach we use, and it was extended and applied to Markov switching models by Carvalho, Johannes, Lopes, and Polson (2010a, 2010b) and Carvalho, Lopes and Polson (2009). Details of the algorithms are given in those papers.

The first step of the approach, data augmentation, introduces a conditional sufficient statistics, $T_{t}$, for the parameters. Sufficient statistics imply that the full posterior distribution of the parameters conditional on entire history of latent states and data takes a known 
functional form conditional on a vector of sufficient statistics: $p\left(\theta \mid s^{t}, y^{t}\right)=p\left(\theta \mid T_{t}\right)$, where $p\left(\theta \mid T_{t}\right)$ is a known distribution. The conditional sufficient statistics are given by $T_{t+1}=$ $\mathcal{T}\left(T_{t}, s_{t+1}, y_{t+1}\right)$, where the function $\mathcal{T}(\cdot)$ is analytically known, which implies the sufficient statistics can be recursively updated. For Markov switching models, the sufficient statistics contain random variables such as the number of times and duration of each state visit, the mean and variance of $y_{t}$ in those visits, etc. This step requires conjugate priors.

The key is that it is easier to sample from $p\left(\theta, T_{t}, s_{t} \mid y^{t}\right)$ than $p\left(\theta, s_{t} \mid y^{t}\right)$, where

$$
p\left(\theta, T_{t}, s_{t} \mid y^{t}\right)=p\left(\theta \mid T_{t}\right) p\left(T_{t}, s_{t} \mid y^{t}\right) .
$$

By the definition of sufficient statistics and the use of conjugate priors, $p\left(\theta \mid T_{t}\right)$ is a known distribution (e.g., normal). This transforms the problem of sequential learning of parameters and states into one of sequential learning of states and sufficient statistics, and then standard updating by drawing from $p\left(\theta \mid T_{t}\right)$. The dimensionality of the target distribution, $p\left(\theta, T_{t}, s_{t} \mid y^{t}\right)$, is fixed as the sample size increases.

An $N$ - particle approximation, $p^{N}\left(\theta, T_{t}, s_{t} \mid y^{t}\right)$, approximates $p\left(\theta, T_{t}, s_{t} \mid y^{t}\right)$ via 'particles' $\left\{\left(\theta, T_{t}, s_{t}\right)^{(i)}\right\}_{i=1}^{N}$ so that:

$$
p^{N}\left(\theta, T_{t}, s_{t} \mid y^{t}\right)=\frac{1}{N} \sum_{i=1}^{N} \delta_{\left(\theta, T_{t}, s_{t}\right)^{(i)}}
$$

where $\delta$ is a Dirac mass. A particle filtering algorithm merely consists of a recursive algorithm for generating new particles, $\left(\theta, T_{t+1}, s_{t+1}\right)^{(i)}$, given existing particles and a new observation, $y_{t+1}$. The approach developed in Johannes and Polson (2008) and Carvalho, Johannes, Lopes, and Polson $(2009 \mathrm{a}, 2009 \mathrm{~b})$ generates a direct or exact sample from $p^{N}\left(\theta, T_{t}, s_{t} \mid y^{t}\right)$, without resorting to importance sampling or other approximate methods. The algorithm is straightforward to code and runs extremely quickly so that it is possible to run for large values $N$, which is required to keep the Monte Carlo error low. These draws can be used to estimate parameters and states variables.

In addition to sequential parameter estimation, particle filters can also be used for Bayesian model comparison. Bayesian model comparison and hypothesis testing utilizes the Bayes factor, essentially a likelihood ratio between competing specifications. Formally, given a number of competing model specifications, generically labeled as model $\mathcal{M}_{k}$ and $\mathcal{M}_{j}$, 
the Bayesian approach computes the probability of model $k$ as:

$$
p\left(\mathcal{M}_{k} \mid y^{t}\right)=\frac{p\left(y^{t} \mid \mathcal{M}_{k}\right) p\left(\mathcal{M}_{k}\right)}{\sum_{j=1}^{N} p\left(y^{t} \mid \mathcal{M}_{j}\right) p\left(\mathcal{M}_{j}\right)},
$$

where $p\left(\mathcal{M}_{k}\right)$ is the prior probability of model $k$,

$$
p\left(y^{t+1} \mid \mathcal{M}_{k}\right)=p\left(y_{t+1} \mid y^{t}, \mathcal{M}_{k}\right) p\left(y^{t-1} \mid \mathcal{M}_{k}\right)
$$

and

$$
p\left(y_{t+1} \mid y^{t}, \mathcal{M}_{i}\right)=\int p\left(y_{t+1} \mid \theta, s_{t}, \mathcal{M}_{i}\right) p\left(\theta, s_{t} \mid y^{t}, \mathcal{M}_{i}\right) d\left(\theta, s_{t}\right)
$$

is the marginal likelihood of observation $y_{t+1}$, given data up to time $t$ in model $k$. Marginal likelihoods are not known analytically and are difficult to compute even using MCMC methods. Since our algorithm provides approximate samples from $p\left(s_{t}, \theta \mid y^{t}\right)$, it is straightforward to estimate marginal likelihoods via

$$
p^{N}\left(y_{t+1} \mid y^{t}, \mathcal{M}_{k}\right)=\frac{1}{N} \sum_{i=1}^{N} p\left(y_{t+1} \mid\left(\theta, s_{t}\right)^{(i)}, \mathcal{M}_{k}\right)
$$

For all of our empirical results, we ran particle filtering algorithms with $N=10 \mathrm{~K}$ particles. We performed extensive simulations to insure that this number of particles insured a low and negligible Monte Carlo error.

\subsection{Priors}

Table 5 shows the prior parameters for the three different models we consider. The historical and look-ahead priors are different along some important dimensions. In particular, preWW2 consumption data is a lot more volatile than the post-war data (annual standard deviation of $4.8 \%$ in the pre-WW2 data versus $1.36 \%$ in post-WW2 data). This has been, in part, attributed to inferior pre-war data that is more noisy and sample that contains a more cyclical component of the economy (Romer, 1989). What is true, nevertheless, is that recessions were more frequent and lasted longer in the pre-WW2 data, and that the Great Depression was a worse recession than ever experienced afterwards, current crisis included. This is reflected in the disaster state in the 3-state models, in particular for the historical prior, akin to the disaster risk considered in Barro (2008). 
For the historical prior, we have estimated, respectively, the 2- and 3-state models starting with very flat priors on the annual Shiller data. The posterior obtained at the end of the pre-war sample is transformed into a prior for the quarterly post-WW2 sample by dividing the average expected means and standard deviations within each regime by 4 , and the average transition probability matrix, $\Pi$, is taken to the power of $1 / 4$. This is of necessity somewhat ad hoc - first, a 2-state model on annual data does not imply a 2-state model on quarterly data; second, one would usually divide standard deviations by 2 to go from annual to quarterly. However, a large fraction of the pre-WW2 excess volatility is likely due to noisy data, which is not what we intend to capture with our prior. What is more, applying priors where the mean belief of the standard deviation of consumption growth within each regime is counter-factually high, leads to a state identification issue: the difference in the average beliefs of the mean within each state is too small relative to the volatilities and so the procedure cannot identify the separate states.

The look-ahead priors have mean values equal to the posterior from the corresponding historical priors in 2009:Q1. These are very close to what would be the maximum likelihood estimates obtained from estimating the 2- and 3-state models using the post-WW2 quarterly sample. The look-ahead priors have lower consumption growth volatility and higher persistence of the good state relative to the historical priors. Thus, the look-ahead prior reflects an expectation in 1947:Q1 of the world having higher growth and lower volatility than in the period before WW2. In terms of the tightness of the priors, the expansion state (always state 1), which has occurred the most, has the tightest priors, the recession state (state 2) has flatter priors as this state is visited less often, while the disaster state (state 3), for the 3 -state models, has the flattest priors. This state is the one agents has the least information about, as it is a rare event.

For the extended model with both consumption and GDP growth, the priors are set to match the consumption-only model as much as possible to minimize the priors' effect on the comparison of the models. Since the means of the hidden state variable are equal to the means of the consumption growth in each state, the priors of these means are the same as in the consumption-only model. We also match the prior means of the total variance of consumption growth with similar flatness. However, since the specification allows for idiosyncratic noise in consumption growth $\left(\sigma_{c} \varepsilon_{t}^{c}\right)$, we set both the mean of the variance of the hidden state variable in each state and the mean of the variance of the noise component to half of the prior mean of the total variance of consumption growth, with similar flatness. This 


\section{APPENDIX: Table 5 - Priors}

Table 5: The table shows the historical and look-ahead priors for the different models considered in the paper. The parameters within a state (mean and variance) have Normal/Inverse Gamma distributed priors, while the transition probabilities have Beta distributed priors. Note that $\hat{\pi}_{i j} \equiv$ $\frac{\pi_{i j}}{1-\pi_{i j}}$.

Historical priors

\begin{tabular}{|c|c|c|c|c|c|c|c|c|}
\hline \multicolumn{3}{|c|}{ Priors for i.i.d. model } & \multicolumn{3}{|c|}{ Priors for 2-state model } & \multicolumn{3}{|c|}{ Priors for 3 -state model } \\
\hline Par. & Mean & St.Dev & Par. & Mean & St.Dev & Par. & Mean & St.Dev \\
\hline$\mu$ & $0.9 \%$ & $0.28 \%$ & $\mu_{1}$ & $1.0 \%$ & $0.25 \%$ & $\mu_{1}$ & $1.0 \%$ & $0.25 \%$ \\
\hline$\mu_{J}$ & $-2.0 \%$ & $1.0 \%$ & $\mu_{2}$ & $-0.5 \%$ & $0.5 \%$ & $\mu_{2}$ & $-0.4 \%$ & $0.5 \%$ \\
\hline$\sigma^{2}$ & $(1.3 \%)^{2}$ & $(1.3 \%)^{2}$ & $\sigma_{1}^{2}$ & $(0.5 \%)^{2}$ & $(0.5 \%)^{2}$ & $\begin{array}{l}\mu_{3} \\
\sigma_{1}^{2}\end{array}$ & $\begin{array}{r}-2.0 \% \\
(0.5 \%)^{2}\end{array}$ & $\begin{array}{r}1.5 \% \\
(0.5 \%)^{2}\end{array}$ \\
\hline$\sigma_{J}^{2}$ & $(2.0 \%)^{2}$ & $(2.0 \%)^{2}$ & $\sigma_{2}^{2}$ & $(1.0 \%)^{2}$ & $(1.0 \%)^{2}$ & $\begin{array}{l}\sigma_{2}^{2} \\
\sigma_{3}^{2}\end{array}$ & $\begin{array}{l}(1.0 \%)^{2} \\
(1.5 \%)^{2}\end{array}$ & $\begin{array}{l}(1.0 \%)^{2} \\
(1.5 \%)^{2}\end{array}$ \\
\hline$\lambda$ & 0.05 & 0.05 & $\pi_{11}$ & 0.95 & 0.034 & $\pi_{11}$ & 0.95 & 0.034 \\
\hline & & & & & & $\hat{\pi}_{12}$ & 0.80 & 0.16 \\
\hline & & & & & & $\hat{\pi}_{21}$ & 0.80 & 0.16 \\
\hline & & & $\pi_{22}$ & 0.80 & 0.16 & $\pi_{22}$ & 0.75 & 0.19 \\
\hline & & & & & & $\hat{\pi}_{31}$ & 0.33 & 0.24 \\
\hline & & & & & & $\pi_{33}$ & 0.40 & 0.20 \\
\hline
\end{tabular}

Look-ahead priors

\begin{tabular}{|c|c|c|c|c|c|c|c|c|}
\hline \multicolumn{3}{|c|}{ Priors for i.i.d. model } & \multicolumn{3}{|c|}{ Priors for 2 -state model } & \multicolumn{3}{|c|}{ Priors for 3 -state model } \\
\hline Par. & Mean & St.Dev & Par. & Mean & St.Dev & Par. & Mean & St.Dev \\
\hline$\mu$ & $0.61 \%$ & $0.12 \%$ & $\mu_{1}$ & $0.68 \%$ & $0.09 \%$ & $\mu_{1}$ & $0.68 \%$ & $0.08 \%$ \\
\hline$\mu_{J}$ & $-1.2 \%$ & $0.23 \%$ & $\mu_{2}$ & $0.2 \%$ & $0.5 \%$ & $\mu_{2}$ & $0.3 \%$ & $0.5 \%$ \\
\hline $\begin{array}{l}\sigma^{2} \\
\sigma_{J}^{2}\end{array}$ & $\begin{array}{l}(0.22 \%)^{2} \\
(0.2 \%)^{2}\end{array}$ & $\begin{array}{l}(0.22 \%)^{2} \\
(0.2 \%)^{2}\end{array}$ & $\begin{array}{l}\sigma_{1}^{2} \\
\sigma_{2}^{2}\end{array}$ & $\begin{array}{r}(0.36 \%)^{2} \\
(0.7 \%)^{2}\end{array}$ & $\begin{array}{c}(0.36 \%)^{2} \\
(0.7 \%)^{2}\end{array}$ & $\begin{array}{l}\mu_{3} \\
\sigma_{1}^{2} \\
\sigma_{2}^{2} \\
\sigma_{3}^{2}\end{array}$ & $\begin{array}{r}-1.14 \% \\
(0.35 \%)^{2} \\
(0.7 \%)^{2} \\
(0.7 \%)^{2}\end{array}$ & $\begin{array}{r}0.5 \% \\
(0.35 \%)^{2} \\
(0.7 \%)^{2} \\
(0.7 \%)^{2}\end{array}$ \\
\hline$\lambda$ & 0.05 & 0.05 & $\pi_{11}$ & 0.95 & 0.034 & $\pi_{11}$ & 0.95 & 0.034 \\
\hline & & & & & & $\hat{\pi}_{12}$ & 0.83 & 0.14 \\
\hline & & & & & & $\hat{\pi}_{21}$ & 0.67 & 0.24 \\
\hline & & & $\pi_{22}$ & 0.80 & 0.16 & $\pi_{22}$ & 0.75 & 0.19 \\
\hline & & & & & & $\hat{\pi}_{31}$ & 0.50 & 0.29 \\
\hline & & & & & & $\pi_{33}$ & 0.33 & 0.24 \\
\hline
\end{tabular}

way, the total prior mean variance of consumption growth, is the same as in the consumption only case. The priors for the transition probabilities are the same as in the consumption only case. For $\alpha$ and $\beta$ in the GDP growth equation, the prior mean is -0.2 for $\alpha$ and 1.2 for 
$\beta$, and prior standard deviation is 0.45 for both. Finally, the prior mean of the idiosyncratic component of the variance of GDP growth is set by matching the variance of the GDP growth in the post-war data.

\subsection{Time-Averaging of Consumption Data and Model Probabili- ties}

The aggregate consumption data is time-averaged, which has implications for the volatility and autocorrelation structure of measured consumption growth. In particular, Working (1960) shows that time-averaging of i.i.d. data leads to lower variance (the variance is decreased by a factor of 1.5) and an autocorrelation of 0.25. Time-averaging can therefore artificially lead us to conclude that consumption growth follows a non-i.i.d. process (e.g., as we would get in the 2-state model with persistent states). Further, Hall (1978) argues theoretically and empirically that consumption growth is close to i.i.d. To ensure the rejection of the i.i.d. model we document in the paper is not an artifact of the time-averaging, we here assume the null hypothesis that consumption growth is in fact i.i.d., and remove the autocorrelation induced by time-averaging by creating the following residuals:

$$
\nu_{c, t}=\Delta c_{t}-0.25 * \Delta c_{t-1}
$$

We then redo the filtration exercise (parameters and models) and assign a prior probability of the i.i.d. model of 0.95. Figure 16 shows that also in this case, even with the strong model prior imposed, the i.i.d. model is rejected by the Bayesian agent about half-way through the sample.

\subsection{Model solution and pricing}

Here we give the details for how the prices of the consumption and aggregate equity claim in Section 4 are computed. At each point in time $t$, we price the equity claim given a set of model parameters, which are set equal to the mean beliefs at the time. The 2-state model and 3-state models have parameters

$$
\begin{aligned}
\theta^{(2)} & =\left\{\mu_{1}, \mu_{2}, \sigma_{1}, \sigma_{2}, \pi_{11}, \pi_{22}\right\}, \\
\theta^{(3)} & =\left\{\mu_{1}, \mu_{2}, \mu_{3}, \sigma_{1}, \sigma_{2}, \sigma_{3}, \pi_{11}, \pi_{12}, \pi_{22}, \pi_{23}, \pi_{13}, \pi_{33}\right\},
\end{aligned}
$$




\section{Figure 16 - Model Probabilities and Time-Averaging of Consumption Data}
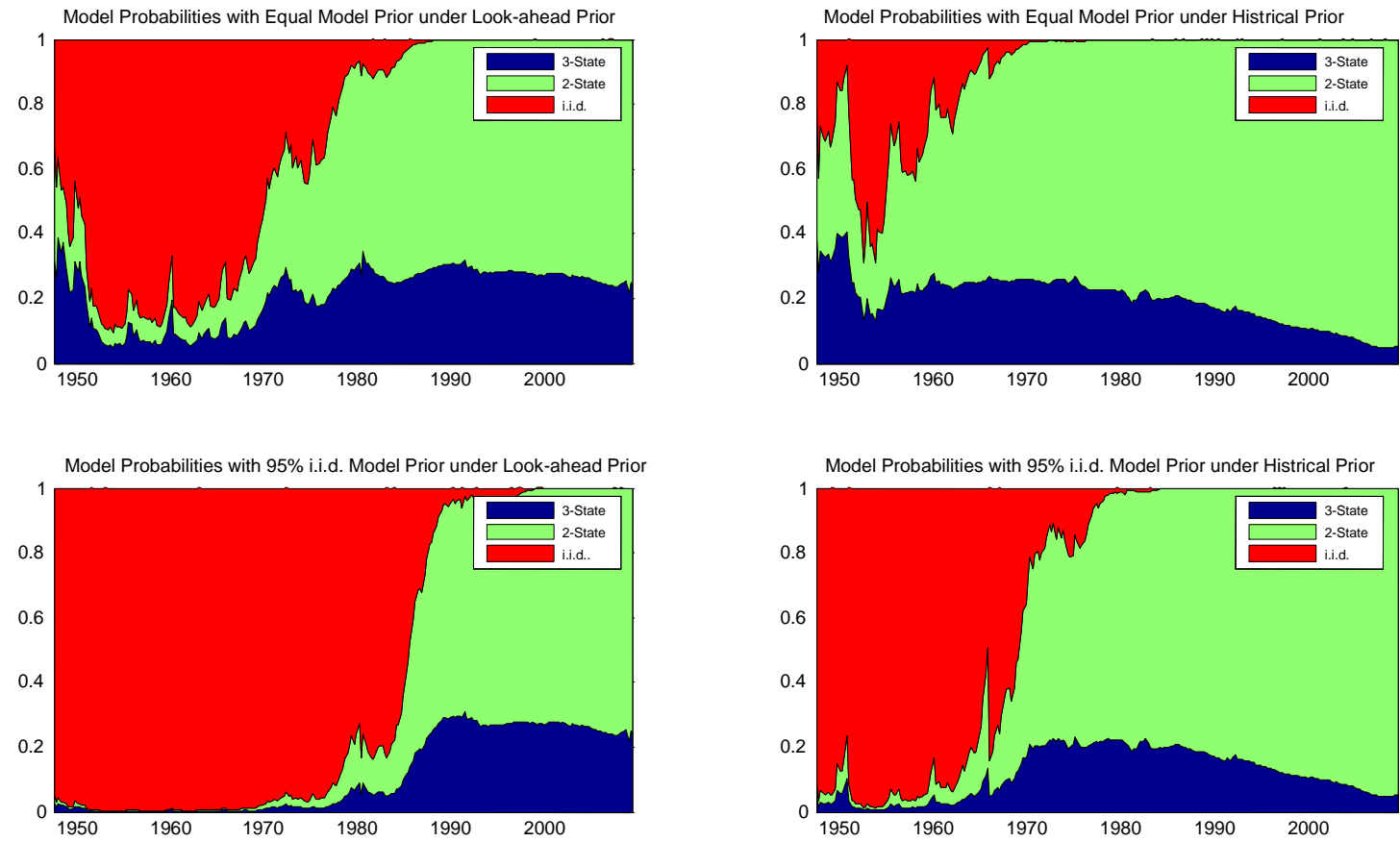

Figure 16: Model probabilities when assuming consumption growth is truly i.i.d. and removing the effect of time-averaging, as calculated by Working (1960). 
respectively. In addition, there is the probability that the 2-state model is the correct model (versus the 3 -state model), which we also set as a constant when the agent prices the equity claim. Denote this probability $p$. Thus, there is a total of 19 parameters that all are estimated using the particle filter and realized consumption (and GDP) data in real time. These parameters will change at each time $t$, but we do not give the parameters time-subscripts to highlight that they are assumed to be constant following the anticipated utility framework in the pricing problem at each time $t$. In addition, there are the preference parameters $\gamma, \psi, \beta$, which are set to the values used in Bansal and Yaron (2004), and the leverage factor $\lambda$ and the idiosyncratic dividend growth volatility $\sigma_{d}$. These parameters remain constant over the sample. When solving for the price-dividend ratio, we can and do ignore the idiosyncratic component of dividend growth.

First, we have to solve for the wealth-consumption ratio, $P C$. At each time $t$, the wealthconsumption ratio is solved using the recursion:

$$
P C\left(s_{t}^{(2)}, \tilde{s}_{t}^{(3)}\right)=\beta E\left[e^{(1-\gamma) \Delta c_{t+1}}\left(1+P C\left(s_{t+1}^{(2)}, \tilde{s}_{t+1}^{(3)}\right)\right)^{\theta} \mid I_{t}\right]^{1 / \theta}
$$

where the wealth-consumption ratio at time $t$ is a function of the state-variables $s_{t}^{(2)}$ and $\tilde{s}_{t}^{(3)}$, and $I_{t}$ is the agent's information set which includes the mean parameter values used as constant parameters, as well as the mean state beliefs. The state-variable $s_{t}^{(2)}$ is the belief that the economy is in state 1 in the 2-state model. Remember that the states are still hidden, even though all the parameters are set to constants, so this belief will have a support of $(0,1)$. Similarly, $\tilde{s}_{t}^{(3)}$ is the $2 \times 1$ vector of state belief probabilities from the 3 -state model - the probability of being in state 1 and the probability of being in state 2 .

In the model solution, the agent updates beliefs about $s^{(2)}$ and $\tilde{s}^{(3)}$ only by observing realized consumption growth - he does not know which model is the true model, or which state is the current state, so this uncertainty must be integrated out in the model solution. Below is a conceptual algorithm for the model solution. ${ }^{26}$

1. Start with an initial guess of the function $P C\left(s^{(2)}, \tilde{s}^{(3)}\right)$ on a grid for the 3 state variables, which all have support $(0,1)$.

2. For each value of $s^{(2)}, \tilde{s}^{(3)}$ on the grid, do points 3. -8 . below:

\footnotetext{
${ }^{26}$ In actually solving the model, we employ numerical integration and not Monte Carlo simulation to find the wealth-consumption ratio. We compute the price-dividend ratio by summing over zero-coupon dividend claims. While we implement the model solution in this way for faster and more accurate model solution, this additional level of detail is not necessary for conceptually understanding how prices are computed.
} 
3. Draw a model (the 2 -state or the 3 -state model) using the model probability $p$.

4. Draw the current state of this model (state 1 , state 2 (or state 3 )), using the state belief for the current values in the grid for $s_{t}^{(2)}$ or $\tilde{s}_{t}^{(3)}$.

5. Given the model and the state, draw a random standard normal shock $\varepsilon_{t+1}$, and compute consumption growth as

$$
\Delta c_{t+1}=\mu_{M, j}+\sigma_{M, j} \varepsilon_{t+1}
$$

where the subscript $M$ refers to the model and the subscript $j$ refers to the state in the same model. The parameters are assumed known and constant as discussed above.

6. Given observed $\log$ consumption growth $\left(\Delta c_{t+1}\right)$ (the agent does not observe the shock $\varepsilon)$ update the agent's belief using Bayes' rule. When finding $s_{t+1}^{(2)}$, condition on the 2-state model being the correct model, and when finding $\tilde{s}_{t+1}^{(3)}$, condition on the 3 -state model being the correct model. See, e.g., Hamilton (1994) for how to update beliefs in switching regime models such as the ones considered here. Note that one has to update the belief for both models $\left(s^{(2)}\right.$ and $\left.\tilde{s}^{(3)}\right)$, even though in the simulation of consumption growth we conditioned on one of the models, as the agent does not know the model.

7. Given $s_{t+1}^{(2)}$ and $\tilde{s}_{t+1}^{(3)}$ and the initial guess for $P C$, we have all we need to evaluate the expression inside the expectation of Equation (11).

8. Repeat 3. -7 . many times and take the average of the different values calculated for the expression inside the expectation of Equation (11). Use this average as an estimate of the expectation in Equation (11). Store the resulting value for $P C\left(s^{(2)}, \tilde{s}^{(3)}\right)$ found for the current place in the grid for $s^{(2)}$ and $\tilde{s}^{(3)}$.

9. Once 3. -8 . has been implemented for all values of $s^{(2)}$ and $\tilde{s}^{(3)}$ on the grid, update the function $P C\left(s^{(2)}, \tilde{s}^{(3)}\right)$.

10. Iterate on 2. - 9. until a suitable convergence criterion for the $P C$ function has been achieved.

Points 1. - 10. gives the wealth consumption ratio at time $t$. The pricing functional $P C\left(s_{t}^{(2)}, \tilde{s}_{t}^{(3)}\right)$ must be computed in this way for each $t$, as the parameters will change at each time $t$. This is the anticipated utility component of the pricing. 
The price-dividend ratio can be found similarly, by iterating on the below expression in the same manner as above for each time $t$ in the sample with its corresponding time $t$ set of parameter values:

$$
P D\left(s_{t}^{(2)}, \tilde{s}_{t}^{(3)}\right)=E\left[\beta^{\theta} e^{(\lambda-\gamma) \Delta c_{t+1}}\left(\frac{P C\left(s_{t+1}^{(2)}, \tilde{s}_{t+1}^{(3)}\right)+1}{P C\left(s_{t}^{(2)}, \tilde{s}_{t}^{(3)}\right)}\right)^{\theta-1}\left(1+P D\left(s_{t+1}^{(2)}, \tilde{s}_{t+1}^{(3)}\right)\right) \mid I_{t}\right] .
$$

Finally, the returns to the equity claim are calculated as follows. For the return from time $t$ to time $t+1$ :

1. Set $s_{t}^{(2)}$ and $\tilde{s}_{t}^{(3)}$ equal to the mean state beliefs at time $t$ (after parameter uncertainty is integrated out).

2. This gives the price dividend ratio at time $t$ as $\frac{P_{t}}{D_{t}}=P D\left(s_{t}^{(2)}, \tilde{s}_{t}^{(3)}\right)$.

3. Set $s_{t+1}^{(2)}$ and $\tilde{s}_{t+1}^{(3)}$ equal to the mean state beliefs at time $t+1$ (after parameter uncertainty is integrated out).

4. This gives the price dividend ratio at time $t+1$ as $\frac{P_{t+1}}{D_{t+1}}=P D\left(s_{t+1}^{(2)}, \tilde{s}_{t+1}^{(3)}\right)$.

5. Next, using realized (in the data) consumption growth, obtain dividend growth as:

$$
\frac{D_{t+1}}{D_{t}}=\left(\frac{C_{t+1}}{C_{t}}\right)^{\lambda} e^{-\frac{1}{2} \sigma_{d}+\sigma_{d} \varepsilon t+1}
$$

where $\varepsilon_{t+1}$ is a draw from a standard normal distribution independent of everything else. These simulated shocks are constrained to have mean zero and variance one over the sample, such that $E_{T}\left[e^{-\frac{1}{2} \sigma_{d . t+1}^{2}+\sigma_{d} \varepsilon_{t+1}}\right]=1$ (in practice, extremely close to 1 ). This is done to ensure that the level of the in-sample average equity return and equity return volatility are not affected by the (by chance) high or low draw of the idiosyncratic component of dividends, or (by chance) high or low volatility of idiosyncratic dividend growth.

6. Given this, the return is calculated as:

$$
R_{t, t+1}=\frac{D_{t+1}}{D_{t}}\left(\frac{P_{t}}{D_{t}}\right)^{-1}\left(1+\frac{P_{t+1}}{D_{t+1}}\right)
$$

